\title{
HALL-HIGMAN TYPE THEOREMS FOR EXCEPTIONAL GROUPS OF LIE TYPE, I
}

\author{
PHAM HUU TIEP AND A. E. ZALESSKI
}

\begin{abstract}
The paper studies the minimum polynomial degrees of $p$-elements in cross-characteristic representations of simple groups of exceptional Lie type whose BN-pair rank is at most 2. Specifically, we prove that the degree in question equals the order of the element.
\end{abstract}

\section{INTRODUCTION}

This paper continues our earlier work [55] devoted to generalize the famous Hall-Higman theorem on the minimum polynomials of $p$ elements in representations of $p$-solvable groups to more general classes of groups. The bulk of the project is the case of almost simple groups. The paper [55] deals mainly with classical groups, and this paper completes the project for quasi-simple groups of BN-pair rank at most 2. Specifically, we prove the following result.

Theorem 1.1. Let $G \in\left\{{ }^{2} B_{2}(q), q>2,{ }^{2} G_{2}(q), q>3,{ }^{2} F_{4}(q), G_{2}(q),{ }^{3} D_{4}(q)\right\}$. Let $g \in G a$ element of prime power order coprime to $q$. Let $\phi$ be a non-trivial irreducible representation of $G$ over a field $F$ of characteristic $\ell$ coprime to $q$. Then the minimum polynomial degree of $\phi(g)$ equals $|g|$, unless possibly when $G={ }^{2} F_{4}(8), \ell=3, p=109$ and $\phi(1)<64692$.

Observe that that it suffices to prove Theorem 1.1 for $F$ algebraically closed. Theorem 1.1 is valid for the Tits group which is a subgroup of index 2 in ${ }^{2} F_{4}(2)$; also see Lemma 7.14 for $G=2 \cdot G_{2}(4)$.

Theorem 1.1 improves our earlier result [55, Theorem 4.6], stating that $\operatorname{deg} \phi(g) \geq|g|(1-1 / p)$ whenever a Sylow $p$-subgroup of $G$ is cyclic.

In some special cases the result of Theorem 1.1 was known earlier. These are

(i) Sylow $p$-subgroups of the quasi-simple group $G$ are cyclic and $\ell \in\{0, p\}$ [60];

(ii) $\ell=0, p>2$ and $G \in\left\{G_{2},{ }^{2} F_{4}(q),{ }^{2} F_{4}(2)^{\prime},{ }^{3} D_{4}(q)\right\}$ [62, Lemmas 4.11 and 4.14];

(iii) $G \cong G_{2}(q), p>2$ and $g$ lies in a parabolic subgroup of $G$ [62, Lemma 4.10].

Notation. Let $G$ be a finite group. Then $|G|$ is the order of $G, Z(G)$ be the center of $G$ and $O_{p}(G)$ the maximal normal $p$-subgroup of $G$ for a prime $p$. We often use $|G|_{p}$ to denote the $p$-part of $|G|$. For $g \in G$ the order of $g$ is denoted by $|g|$ and $o(g)$ is the order of $g$ modulo $Z(G)$. A $p^{\prime}$-element is one of order coprime to $p$.

$\mathbb{F}_{q}$ means the finite field of $q$ elements, $\overline{\mathbb{F}}_{q}$ its algebraic closure and $\mathbb{Q}, \mathbb{C}$ are the rational and complex number fields, respectively. $\mathbb{Z}$ denotes the set of integers.

Let $F$ be an algebraically closed field of characteristic $\ell$, and $\phi$ an $F$-representation of $G$. Then $\operatorname{deg} \phi(g)$ denotes the minimum polynomial degree of $\phi(g)$. We write $\phi \in \operatorname{Irr}_{\ell} G$ to indicate that $\phi$ is irreducible, and use this notation for the Brauer character of $\phi$ too. If $\ell=0$, we drop the subscript $\ell$. If $\chi$ is an ordinary (generalized) character of $G$, and with $\ell$ a fixed prime, then $\chi^{\circ}$ is the restriction of $\chi$ to $\ell^{\prime}$-elements.

2020 Mathematics Subject Classification. 20C15, 20C20, 20C33, 20G05, 20 G40.

Key words and phrases. Finite simple groups of Lie type, cross-characteristic representations, minimum polynomials, $p$-elements, Hall-Higman type theorems.

The first author gratefully acknowledges the support of the NSF (grant DMS-1840702), the Joshua Barlaz Chair in Mathematics, and the Charles Simonyi Endowment at the Institute for Advanced Study (Princeton). 
We denote by $1_{G}$ the trivial character of $G$ (both ordinary and $\ell$-modular), and by $\rho_{G}^{\text {reg }}$ the regular representation of $G$ or the (Brauer) character of it. The ordinary Steinberg representation and its character of a group of Lie type is denoted by $\mathrm{St}_{G}$ or St.

A Brauer character $\phi \in \operatorname{Irr}_{\ell} G$ is called liftable if there exists an ordinary character $\tau$ of $G$ such that $\tau^{\circ}=\phi$. An irreducible representation or $F G$-module is called liftable if the Brauer character of it is liftable.

If $H$ is a subgroup of $G$ and $\eta$ is a character or representation of $H$ then $\tau^{G}$ denotes the induced character. If $\phi$ is a character or representation of $G$ then $\left.\phi\right|_{H}$ stands for the restriction of $\phi$ to $H$.

If $V$ is an $F G$-module and $X$ a subset of $G$ we write $V^{X}$ or $C_{V}(X)$ for the subspaces of elements of $V$ fixed by all $x \in X$.

For integers $a, b>0$ we write $(a, b)$ for the greatest common divisor of $a, b$ and $a \mid b$ means that $b$ is a multiple of $a$. We also write $(\chi, \phi)$ for the inner product of characters $\chi, \phi$ of a group $G$. We write $\operatorname{diag}\left(x_{1}, \ldots, x_{m}\right)$ for a block-diagonal matrix with diagonal blocks $x_{1}, \ldots, x_{m}$.

A finite group of Lie type is that of shape $\mathbf{G}^{\mathrm{F}}$, where $\mathbf{G}$ is a reductive algebraic group and $\mathrm{F}$ a Frobenius endomorphism of it. For more information see [4] or [10].

\section{Preliminaries}

Lemma 2.1. Let $C$ be a cyclic group of order coprime to $\ell$, and $\chi$ a non-trivial Brauer character of $C$ such that $\chi(c)=a$ for every $1 \neq c \in C$ (so $\chi$ is constant on $C \backslash\{1\})$. Then $\chi=$ $\frac{\chi(1)-a}{|C|} \cdot \rho_{C}^{\mathrm{reg}}+a \cdot 1_{C}$. In particular, if $a \geq 0$ or $a<0$ and $-a \cdot(|C|-1)<\chi(1)$ then $\chi=\rho_{C}^{\mathrm{reg}}+\chi^{\prime}$ for some proper character $\chi^{\prime}$ of $C$.

Proof. The first claim is obvious as $\chi-a \cdot 1_{G}$ vanishes at $C \backslash\{1\}$. Let $a \geq 0$; then $\frac{\chi(1)-a}{|C|}>0$ as this equals to the multiplicity of any non-trivial irreducible character of $C$ in $\chi$. Let $0<-a<|C|$. Then $\frac{\chi(1)-a}{|C|} \cdot \rho_{C}^{\mathrm{reg}}+a \cdot 1_{C}=\frac{\chi(1)+a(|C|-1)}{|C|} \cdot \rho_{C}^{\mathrm{reg}}-a \cdot \rho_{C}^{\mathrm{reg}}+a \cdot 1_{C}$, so $\chi(1)+a(|C|-1)>0$ implies the second claim in this case.

Lemma 2.2. [32, Lemma IX.2.7] Let $p, r$ be primes and $a, b$ positive integers such that $p^{a}=$ $r^{b}+1$. Then either

(i) $p=2, b=1$, and $r$ is a Mersenne prime, or

(ii) $r=2, a=1$, and $p$ is a Fermat prime, or

(iii) $p^{a}=9$.

Lemma 2.3. [32, Theorem 1.10] Let $G=A H$ be a semidirect product, where $A$ is an abelian normal subgroup of $G$ and $H=\langle h\rangle$ is a p-group. Let $\phi$ be a faithful $\ell$-representation of $G$. Suppose that $(p \ell,|A|)=1$ and $C_{A}(h)=Z(G)$. Then $\operatorname{deg} \phi(h)=o(h)$.

Corollary 2.4. Let $\mathrm{SL}_{n}(q) \leq G \leq \mathrm{GL}_{n}(q)$ and let $h \in G$ be a non-central p-element. Let $\phi$ be an $\ell$-modular representation of $G$ such that $\operatorname{ker} \phi \leq Z(G)$ and $\ell \nmid q$. Suppose that $p \nmid q$ and $h$ is not irreducible on the natural $\mathrm{GL}_{n}(q)$-module $\mathbb{F}_{q}^{n}$. Then $\operatorname{deg} \phi(h)=o(h)$.

Proof. Let $V=\mathbb{F}_{q}^{n}$ and let $W \neq 0$ be a proper $h$-stable subspace of $V$. Let

$$
A:=\{g \in G \mid g W=W \text { and } g \text { acts trivially on both } W \text { and } V / W\} .
$$

Then $A$ is an abelian group, $(|A|, p \ell)=1$ and $h A h^{-1}=A$. In addition, $C_{A}(h) \leq Z(G)$ (in fact $\left.C_{\mathrm{GL}_{n}(q)}(A)=A Z(G)\right)$. So the result follows from Lemma 2.3 .

Lemma 2.5. Let $G=\operatorname{Sp}_{2 n}(q)$, $q$ even, $n>1$, and let $g \in G$ be a reducible $p$-element for $p \nmid \backslash q$. Let $\phi \in \operatorname{Irr}_{\ell} G$ with $\operatorname{dim} \phi>1$ and $l \neq 2$. Then $\operatorname{deg} \phi(g)=|g|$.

Proof. If $g$ belongs to a parabolic subgroup of $G$ then the result is contained in [11. Otherwise, $|g|>3$ and $g \in H \cong H_{1} \times H_{2}$, where $H_{1} \cong \operatorname{Sp}_{2 k}(q), H_{2} \cong \operatorname{Sp}_{2 l}(q), k+l=n$. Then $g=g_{1} g_{2}$, where $g_{1} \in H_{1}, g_{2} \in H_{2}$ are $p$-elements. We may assume that $|g|=\left|g_{1}\right|$ and moreover that $g_{1}$ is irreducible in $H_{1}$. In addition, we may assume that $(k, q) \neq(1,2)$ as otherwise $|g|=3$. 
By [55, Corollary 3.8], the restriction $\left.\phi\right|_{H}$ contains an irreducible constituent $\tau=\tau_{1} \otimes \tau_{2}$ $\left(\tau_{1} \in \operatorname{Irr}_{\ell} H_{1}, \tau_{2} \in \operatorname{Irr}_{\ell} H_{2}\right)$ such that both $\operatorname{dim} \tau_{1}, \operatorname{dim} \tau_{2}>1$. Then $\tau(g)=\tau_{1}\left(g_{1}\right) \otimes \tau_{2}\left(g_{2}\right)$. If $\operatorname{deg} \tau_{1}\left(g_{1}\right)=\left|g_{1}\right|$ then we are done. Otherwise, by [55, Lemma 3.3 and Prop. 5.7], $\left|g_{1}\right|=q^{k}+1$ and $\operatorname{deg} \tau_{1}\left(g_{1}\right) \geq\left|g_{1}\right|-2$ (in fact, $\operatorname{deg} \tau_{1}\left(g_{1}\right) \geq\left|g_{1}\right|-1$ if $k>1$ ). By Lemma 2.2, $\left|g_{1}\right|=q^{k}+1$ implies either $\left|g_{1}\right|=p=q^{k}+1$ or $q^{k}=8,\left|g_{1}\right|=9$.

Suppose first that $\left|g_{1}\right|=p>3$. Then $\operatorname{deg} \tau_{2}\left(g_{2}\right) \geq 3$ by [55, Theorem 1.2], and hence $\operatorname{deg} \tau(g)=p$ by [55, Lemma 2.12].

Suppose that $|g|=9, q=8$. Then $\operatorname{deg} \tau_{2}\left(g_{2}\right) \geq 3$ and $\operatorname{deg} \tau_{1}\left(g_{1}\right) \geq 7$. If $\ell>3$ then [55, Lemma 2.12(i)] again yields the result. Let $\ell=3$. Let $J_{i}$ denote the Jordan block of size $i$ over $\mathbb{F}_{3}$. Then the minimum polynomial degree of $J_{7} \otimes J_{3}$ equals 9 [53, Lemma 2.11]); in addition, if $a \geq 7, b \geq 3$ then the minimum polynomial degree of $J_{a} \otimes J_{b}$ is at least 9 [53, Lemma 2.10]. Therefore, $\operatorname{deg} \tau(g)=9$.

Let $q=2, k=3$ and $|g|=9$. Then $\operatorname{deg} \tau_{1}\left(g_{1}\right) \geq 7$ by [55, Prop. 5.7(ii)]. If $\operatorname{deg} \tau_{2}\left(g_{2}\right) \geq 3$, then the result follows as above. Suppose that $\operatorname{deg} \tau_{2}\left(g_{2}\right)=2$. Then $\left|g_{2}\right|=3$. If $H_{2} \neq \mathrm{Sp}_{2}(2)$ then $g$ is contained in a parabolic subgroup of $G$. So $l=2, n=4$. In this case we show that $\left.\phi\right|_{H_{1}}$ has an irreducible constituent of degree greater than 7 . Indeed, $H_{1}$ is contains in a parabolic subgroup $P$, the stabilizer of a line at the natural $\mathbb{F}_{2} G$-module. Let $Q=O_{2}(P)$. Then $Q$ is an abelian group. Then $\left.\phi\right|_{Q}$ is a direct sum of linear representations $\lambda$ of $Q$ permuted by $P$ when $P$ acts on $Q$ by conjugation. Then there is $\lambda$ whose $\left\langle g_{1}\right\rangle$-orbit is faithful. Let $\Lambda=H_{1} \lambda$ be the $H_{1}$-orbit of $\lambda$ with point stabilizer $C_{H_{1}}(\lambda) \cong P_{1}$, where $P_{1}$ is the stabilizer of a vector at the natural $\mathbb{F}_{2} H_{1}$-module. This yields a permutational $F H_{1}$-module $L \cong 1_{P_{1}}^{H_{1}}$. The composition factors of $L$ are the reduction modulo 3 of those for the similar module over the complex numbers. The latter decomposes as $1_{H_{1}}+\chi_{1}+\chi_{2}$, where $\chi_{1}(1)=27$ and $\chi_{2}(1)=35\left[\underline{6}\right.$, p. 46]. As $\chi_{1}^{\circ}$, the restriction of $\chi$ to $3^{\prime}$-elements, coincides with the Brauer character of $H_{1}$ of degree 27 [33], we conclude that $L$ has an irreducible constituent of degree 27. This contradicts [55, Prop. 5.7(ii)].

Lemma 2.6. Let $G=\mathrm{SL}_{3}(q), q>2$, and let $\phi \in \operatorname{Irr}_{F}(G), \operatorname{dim} \phi>1, \ell \nmid q$. Let $g \in G$ be a $p$-element. Then either $\operatorname{deg} \phi(g)=o(g)$, or $(3, q-1)=1,|g|=q^{2}+q+1$; moreover, $\operatorname{dim} \phi=q^{2}+q-1$ if $p=\ell$, whereas if $p \neq \ell$ then $\operatorname{deg} \phi(g)=\operatorname{dim} \phi=q^{2}+q$ and 1 is not an eigenvalue of $\phi(g)$.

Proof. If $g$ is reducible in $G$ then the result follows from Corollary 2.4. Suppose that $g$ is irreducible in $G$, and hence $|g|$ divides $q^{2}+q+1$. Observe that $p>2$ as $q^{2}+q+1$ is odd. If Sylow $p$-subgroups are not cyclic then $p=3$ and $3 \mid(q-1)$, and then $g$ is reducible by [62, Lemma 3.2] a contradiction.

So Sylow $p$-subgroups are cyclic. If $\ell=0$ or $p$ then the result is a special case of [60, Theorem 1.1], and the claim on eigenvalue 1 for $\ell=0$ is contained in [60, Corollary 1.3(4)]. Let $\ell \neq p$. According to [55, Example 3.2(ii)], either $\phi$ lifts to characteristic 0 and the result follows from that for $\ell=0$, or $\ell$ divides $q^{2}+q+1$ and the Brauer character of $\phi$ coincides on the $\ell^{\prime}$-elements with $\tau-1_{G}$, where $\tau$ is the unipotent character of degree $q^{2}+q$ of $G$. This again implies the result.

Lemma 2.7. (Borel and Tits, see [21, §13.1]) Let $H$ be a finite reductive group in characteristic $r$ and $g \in G$. If $g$ normalizes an $r$-subgroup of $H$ then $g$ belongs to a parabolic subgroup of $H$. In particular, this holds if $g$ is not regular.

Let $G$ be a finite quasi-simple group of Lie type in characteristic $r>0$. Let $\Phi_{m}(x)$ denote the cyclotomic polynomial for $m$-th roots of 1 , and $\prod_{m} \Phi_{m}^{l_{m}}(x)$ a polynomial associated with $G$, see [21], pages $110--111$. Set $|G|_{r^{\prime}}:=|G| /|U|$ where $U$ is a Sylow $r$-subgroup of $G$. Then $|G|_{r^{\prime}}=\prod_{m} \Phi_{m}^{l_{m}}(q)$. If $G={ }^{2} B_{2}(q),{ }^{2} F_{4}(q)$ we assume that $q=2^{2 a+1}$, and if $G={ }^{2} G_{2}(q)$ then $q=3^{2 a+1}$ which notation agrees with that in [21. Throughout this section $m_{p}$ denotes the multiplicative order of $q(\bmod p)$, and $e_{p}$ is the $p$-part of $\Phi_{m_{p}}(q)$. Observe that $\Phi_{1}(q)=q-1$, $\Phi_{2}(q)=q+1, \Phi_{3}(q)=q^{2}+q+1, \Phi_{4}(q)=q^{2}+1, \Phi_{5}(q)=q^{4}+q^{3}+q^{2}+q+1, \Phi_{6}(q)=q^{2}-q+1$, $\Phi_{8}(q)=q^{4}+1, \Phi_{10}(q)=q^{4}-q^{3}+q^{2}-q+1, \Phi_{12}(q)=q^{4}-q^{2}+1$. 
Lemma 2.8. ([22, §4.10.2] and [2])

(i) $|G|_{r^{\prime}}=\prod_{m} \Phi_{m}^{l_{m}}(q)$;

(ii) Then $S$ is cyclic if and only if there is exactly one $m$ such that $p$ divides $\Phi_{m}(q)$ and $l_{m}=1$ for this $m$.

(iii) For every factor $\Phi_{m}(x)$ of the above polynomial there is a torus $T$ of $G$ such that $|T|=$ $\Phi_{m}^{l_{m}}(q)$. All tori of order $\Phi_{m}^{l_{m}}(q)$ are conjugate in $G$. In addition, $T$ is a direct product of subtori of order $\Phi_{m}(q)$.

(iv) Let $m_{p}$ be the multiplicative order of $q(\bmod p)$ and let $T_{p}$ be a torus in (iii) corresponding to $m=m_{p}$. Then $N_{G}(T)$ contains a conjugate of $S$. Furthermore, if $S \subset N_{G}(T)$ then the subgroup $A:=T \cap S$ is homocyclic of rank $l_{m_{p}}$ and of exponent $e_{p}$.

Lemma 2.9. Let $\mathbf{G}$ be a simple simply connected algebraic group of rank $n>0, F$ a Frobenius endomorphism of $\mathbf{G}$, and $G:=\mathbf{G}^{\mathrm{F}}$. Let $A$ be as in Lemma 2.8(iv).

(i) [62, Proposition 4.8] Let $p>2$ be a prime dividing $|G|$ and $e_{p}=:\left|\Phi_{m_{p}}(q)\right|_{p}$, that is, $e_{p}$ is the exponent of $A$. Then every p-element $g \in G$ of order at most $e_{p}$ is conjugate to an element in $A$.

(ii) Let $\varepsilon \in\{ \pm 1\}$ be such that $4 \mid(q-\varepsilon)$, and let $q-\varepsilon=2^{e} m$, where $m$ is odd. Suppose that $G$ has a maximal torus $T$ of order $(q-\varepsilon)^{n}$. Then every 2-element of $G$ of order at most $2^{e}$ is conjugate to an element of $T$.

Proof. (ii) Let $\mathbf{T}$ be an F-stable maximal torus of $\mathbf{G}$ such that $T=\mathbf{T}^{\mathrm{F}}$. Let $g \in G$ with $g^{2^{e}}=1$. It is well-known that $g$ is $\mathbf{G}$-conjugate to an element $g^{\prime} \in \mathbf{T}$. Set $T_{2}=\left\{t \in \mathbf{T}: t^{2^{e}}=1\right\}$. Then $\left|T_{2}\right|=2^{e n}$. Therefore, $T_{2}$ coincides with the subgroup $\left\{x \in T: x^{2^{e}}=1\right\}$, so $g^{\prime} \in T$. As $\mathbf{G}$ is simply connected, $C_{\mathbf{G}}(g)$ is connected [52, Ch.II, 3.9]. By [52, Ch.I, 3.4], the elements $g, g^{\prime} \in G$ are conjugate in $G$ provided $C_{\mathbf{G}}(g)$ is connected. So the claim follows.

\section{Some observations on Representations of groups of Lie type}

Recall that $\operatorname{Irr}(G)$ partitions into (rational) Lusztig series denoted by $\mathcal{E}_{s}$, where $s$ runs over the representatives of the conjugacy classes of semisimple elements of the dual group $G^{*}$. The characters in $\mathcal{E}_{1}$ are called unipotent.

Lemma 3.1. Let $T$ be a maximal torus of a finite reductive group $G=\mathbf{G}^{F}$, and let $t_{1}, t_{2} \in T$ be regular elements. If $t_{1}, t_{2}$ are conjugate in $G$ then they are conjugate in $N_{G}(T)$.

Proof. Let $\mathbf{T}$ be the maximal torus of $\mathbf{G}$ containing $T$. Then $\mathbf{T}$ is unique and $t_{1}, t_{2}$ are conjugate in $N_{\mathbf{G}}(\mathbf{T})$. Let $n t_{1} n^{-1}=t_{2}$ with $n \in N_{\mathbf{G}}(\mathbf{T})$. Then $\mathrm{F}(n) t_{1} \mathrm{~F}\left(n^{-1}\right)=t_{2}$, whence $n^{-1} \mathrm{~F}(n) t_{1} n \mathrm{~F}\left(n^{-1}\right)=t_{1}$, that is, $n^{-1} \mathrm{~F}(n) \in C_{\mathbf{G}}\left(t_{1}\right)=\mathbf{T}$. By the Lang theorem, $n^{-1} \mathrm{~F}(n)=$ $t^{-1} \mathrm{~F}(t)$ for some $t \in \mathbf{T}$. So $t n^{-1}=\mathrm{F}(t) \mathrm{F}\left(n^{-1}\right)=\mathrm{F}\left(t n^{-1}\right)$, so $t n^{-1} \in G$ and $x:=n t^{-1} \in G$. Clearly, $x t_{1} x^{-1}=t_{2}$ and $x \in N_{\mathbf{G}}(\mathbf{T}) \cap G=N_{G}(\mathbf{T})$. As $T=\mathbf{T} \cap G$, we have $x T x^{-1}=T$, as required.

Lemma 3.2. Let $G$ be a finite group of Lie type. Let $\chi$ be an irreducible unipotent character of $G$, and $S$ a maximal torus of $G$. Suppose that every element $1 \neq t \in S$ is regular (so $G$ is simple). Then $\chi$ is constant on $S \backslash\{1\}$ and $\chi(t) \in\{0,1,-1\}$. Equivalently, $\left.\chi\right|_{S}=\frac{\chi(1)-\eta}{|S|} \cdot \rho_{S}^{\mathrm{reg}}+\eta \cdot 1_{S}$, where $\eta \in\{0,1,-1\}$.

Proof. For a function $f$ of $G$ denote by $f^{\#}$ the restriction of $f$ to the set of semisimple elements of $G$. By the Deligne-Lusztig theory, if $\chi \in \operatorname{Irr}(G)$ then $\chi^{\#}$ is a $\mathbb{Q}$-linear combination of $R_{T_{i}, \theta_{i}}^{\#}$, where $R_{T_{i}, \theta_{i}}$ are some Deligne-Lusztig characters, $T_{i}$ is a maximal torus of $G$ and $\theta_{i}$ is a linear character of $T_{i}$. Let $a_{i}$ be the coefficient of $R_{T_{i}, \theta_{i}}^{\#}$ in the expression in question. The values $R_{T_{i}, \theta_{i}}(h)$ at the semisimple elements $h \in G$ are given by the formula $R_{T_{i}, \theta_{i}}(h)=\varepsilon\left(T_{i}\right) \varepsilon(G) \theta_{i}^{G}(h) / \operatorname{St}(h)$, where St is the Steinberg character of $G$ and $\varepsilon\left(T_{i}\right), \varepsilon(G) \in\{ \pm 1\}$, see for instance [4, Prop. 7.5.4]. It is well known that a regular semisimple element of $G$ lies in a unique maximal torus, so either 
$T_{i}$ is conjugate to $S$ or $R_{T_{i}, \theta_{i}}(t)=0$ for every $t \in(S \backslash\{1\})$. Therefore, we conclude that either $\chi(t)=0$ for all $t \in(S \backslash\{1\})$, or $\chi(t)=\sum a_{i} R_{S, \theta_{i}}(t)$, with some non-zero coefficient $a_{i}$. (Hence $\chi=\sum a_{i} R_{S, \theta_{i}}+f$, where $f$ is a class function vanishing on $S \backslash\{1\}$.) Furthermore, $\operatorname{St}(h)=\varepsilon\left(T_{i}\right)$ whenever $h$ is regular and $h \in T_{i}$. So $R_{S, \theta_{i}}(t)=\varepsilon(G) \theta_{i}^{G}(t)$, and hence $\chi(t)=\varepsilon(G) \cdot \sum a_{i} \theta_{i}^{G}(t)$. Furthermore, if $h, h^{\prime} \in S$ are conjugate in $G$ and regular then $h, h^{\prime}$ are conjugate in $N=N_{G}(S)$ by Lemma 3.1. As $C_{G}(t)=S$, it follows that $\theta_{i}^{G}(t)=\theta_{i}^{N}(t)$.

If $\chi$ vanishes on $S \backslash\{1\}$ then $\left.\chi\right|_{S}$ is a multiple of the regular character $\rho_{S}^{\text {reg }}$.

Suppose that $\chi$ is unipotent. Then $\theta_{i}=1_{S}$ is the trivial character of $S$, so $\chi(t)=\left(\sum a_{i}\right) \varepsilon(G)$. $1_{S}^{G}(t)=a \varepsilon(G)|N / S|$, where $a=\sum a_{i}$. In particular, $\chi$ is constant on $S \backslash\{1\}$.

Let $p$ be a prime dividing $|S|$. Then $S$ contains a Sylow $p$-subgroup of $G$. As $C_{G}(t)=S$ for $1 \neq t \in S$, every $p$-singular element is conjugate to that in $S$. Therefore, $\chi$ is constant at the $p$-singular elements of $G$. Then, by [46, Theorem 1.3], $\chi$ belongs to the principal $p$-block of $G$ and $\chi(t)=\eta \in\{ \pm 1\}$. Therefore, $\chi(t)=\eta$ for every $1 \neq t \in S$. So we are done in this case.

Remark 3.3. Suppose that $\chi$ is not unipotent. Then $\theta_{i} \neq 1_{T_{i}}$. Then $\theta_{i}^{G}(h)$ is the sum of $\theta_{i}\left(h^{\prime}\right)$, where $h^{\prime}$ runs over all elements of $T_{i}$ that are conjugate to $h$. The number of them is $N_{G}\left(T_{i}\right) / T_{i}$ as $T_{i}$ is a TI-set, and this does not depend on the choice of $1 \neq h \in T_{i}$. Then $\theta_{i}^{G}(h)$ is the sum of $\left|N_{G}\left(T_{i}\right) / T_{i}\right|$ non-trivial $|h|$-roots of unity.

An irreducible Brauer character $\phi$ for $\ell$ different from the defining characteristic of $G$ is called unipotent if $\phi$ is a constituent of $\chi^{\circ}$ for some unipotent ordinary character $\chi$. Let $G^{*}$ be the group dual to $G$. For a semisimple $\ell^{\prime}$-element $s \in G^{*}$, denote by $\mathcal{E}_{\ell, s}$ the union of the sets $\mathcal{E}_{y s}$, where $y \in G^{*}, y s=s y$ and $|y|$ is an $\ell$-power. Then $\mathcal{E}_{\ell, s}$ is a union of $\ell$-blocks ([8, Theorem 9.4.6]), so for every $\phi \in \operatorname{Irr}_{\ell} G$ there exists a semisimple $\ell^{\prime}$-element $s \in G^{*}$ such that $\phi$ is a constituent of $\chi^{\circ}$ for some $\chi \in \mathcal{E}_{\ell, s}$. (Moreover, $\chi$ can be chosen in $\mathcal{E}_{s}$ [27, Theorem 3.1].) Therefore, it is meaningful to write $\phi \in \mathcal{E}_{\ell, s}$.

Lemma 3.4. Let $\phi \in \mathcal{E}_{\ell, s}$ be a Brauer character. Then the restriction of $\phi$ to semisimple $\ell^{\prime}$-elements is a $\mathbb{Q}$-linear combination of the ordinary characters of $\mathcal{E}_{s}$ restricted to semisimple $\ell^{\prime}$-elements.

Proof. Let $\chi \in \mathcal{E}_{s}$ be such that $\phi$ is a constituent of $\chi^{\circ}$. Every irreducible Brauer character of an $\ell$-block is a $\mathbb{Z}$-linear combination of the ordinary characters of this block restricted to $\ell^{\prime}$-elements [44. Lemma 3.16], so $\phi$ is a $\mathbb{Z}$-linear combination of the ordinary characters of $\mathcal{E}_{\ell, s}$ restricted to $\ell^{\prime}$-elements. In turn, the ordinary characters of $\mathcal{E}_{\ell, s}$ restricted to the semisimple elements are $\mathbb{Q}$-linear combinations of the Deligne-Lusztig characters defining $\mathcal{E}_{\ell, s}$ restricted to semisimple elements (see [55, Lemma 4.1]). Therefore, $\phi$ is a $\mathbb{Q}$-linear combination of such Deligne-Lusztig characters restricted to semisimple $\ell^{\prime}$-elements. As every Deligne-Lusztig character from $\mathcal{E}_{y s}$ for $y \in C_{G}(s)$, ys $\neq 1$, restricted to semisimple elements coincides with some Deligne-Lusztig character from $\mathcal{E}_{s}$ restricted to semisimple elements [27, Prop 2.2], $\phi$ is a $\mathbb{Q}$-linear combination of the ordinary characters of $\mathcal{E}_{s}$ restricted to semisimple $\ell^{\prime}$-elements, the result follows.

Remark 3.5. There is a conjecture that $\phi$ is a $\mathbb{Z}$-linear combination of the ordinary characters of $\mathcal{E}_{s}$ restricted to $\ell^{\prime}$-elements. This has been proven for many cases, see [20, Theorem 5.1] and [8. Section 9], in particular, this is true if $G={ }^{2} F_{4}(q)$ and ${ }^{3} D_{4}(q)$, and if $G=S L_{3}(q)$, $\operatorname{SU}_{3}(q)$ for $\ell \nmid|Z(G)|$.

Corollary 3.6. Under the assumptions of Lemma 3.2 let $\phi$ be a unipotent $\ell$-Brauer character of $G$. Then $\phi$ is constant on the set $S \backslash\{1\}$.

Proof. This follows from Lemmas 3.2 and 3.4 .

The following lemma refines Theorem 4.2 in [55].

Lemma 3.7. Under the assumptions of Lemma 3.2 let $\phi \in \operatorname{Irr}_{\ell} G$ and $\phi \in \mathcal{E}_{\ell, s}$. Then one of the following holds: 
(i) $s=1, \phi$ is unipotent and constant on the $\ell^{\prime}$-elements of $S \backslash\{1\}$;

(ii) $s \neq 1,|s|$ is coprime to $|S|$ and $\phi(t)=0$ for all $\ell^{\prime}$-elements $t \in(S \backslash\{1\})$;

(iii) $s \neq 1,|s|$ divides $|S|$ and $\phi$ lifts to characteristic 0 .

Proof. If $s=1$ then $\phi$ is unipotent, and we have (i) by Corollary 3.6. Let $s \neq 1$.

Suppose first that $|s|$ is coprime to $|S|$. Let $\chi \in \mathcal{E}_{s}$. Then, on restriction to semisimple elements, $\chi$ agrees with a $\mathbb{Q}$-linear combination of the Deligne-Lusztig characters $R_{T_{i}, \theta_{i}}$ defining $\mathcal{E}_{s}$ (see [55, Lemma 4.1]). Here, $\left|\theta_{i}\right|=|s|$ by [27, Lemma 2.1(a)], so $|s|$ divides $\left|T_{i}\right|$. Therefore, $T_{i}$ is not conjugate to $S$, and hence $R_{T_{i}, \theta_{i}}(t)=0$ for every $1 \neq t \in S$. So $\chi(s)=0$ for every $\chi \in \mathcal{E}_{s}$. Now (ii) follows by Lemma 3.4 .

Suppose that $|s| \neq 1$ divides $|S|$. As ys is a regular semisimple element for $y \in C_{G}(s)$, ys $\neq 1$, and hence $S=C_{G}(y s)$, the set $\mathcal{E}_{y s}$ consists of a single character of degree $d=|G|_{r^{\prime}} /|S|$, where $r$ is the defining characteristic of $G$. In addition, $\mathcal{E}_{\ell, s}$ is a union of $\ell$-blocks, so, by [44, Lemma 3.16], every irreducible Brauer character $\phi$ in any of these blocks is a $\mathbb{Z}$-linear combination of ordinary characters of degree $d$, and $\phi$ itself is a constituent of $\eta^{\circ}$ for some irreducible character $\eta \in \mathcal{E}_{\ell, s}$, which is of degree $d$. Hence $\phi(1)=d$ and $\phi=\eta^{\circ}$.

\section{Unipotent ELEMENTS IN $\operatorname{GL}_{n}(F)$, Char $F=2$}

Let $1 \neq g \in \mathrm{GL}_{n}(2)=\mathrm{GL}(V)$ be a 2 -element and $z \in\langle g\rangle$ an involution. We set

$$
j(g)=j(z):=\operatorname{dim}(\operatorname{Id}-z) V .
$$

Note that $j(g)$ equals the number of blocks of size 2 in the Jordan canonical form of $z$.

Lemma 4.1. Let $g \in \mathrm{GL}_{n}(2)$ be an element of order $2^{m+1}, m>0$, and $z:=g^{2^{m}}$. Let $J(g)=\left(J_{n_{1}}, \ldots, J_{n_{k}}\right)$ be the Jordan canonical form of $g$. Set $l_{i}=\max \left(0, n_{i}-2^{m}\right)$ for $i=1, \ldots, k$. Then $j(g)=\sum l_{i}$.

Proof. It suffices to prove the statement in the case $k=1$, where we have

$$
j(g)=\operatorname{dim}(\operatorname{Id}-z) V=\operatorname{dim}(\operatorname{Id}-g)^{2^{m}}(V)=n-2^{m}=l_{1} .
$$

Lemma 4.2. Let $0<c<d<n$ be integers, and $n=k d+l$ for $0 \leq l<d$. For a sequence $\lambda=\left(n_{1} \geq \cdots \geq n_{k} \geq 0\right)$ set $\bar{\lambda}=\left(l_{1}, \ldots, l_{k}\right)$ where $l_{i}=\max \left(0, n_{i}-c\right)$ for $i=1, \ldots, k$. Suppose that $\sum n_{i}=n$. Then

$$
\sum_{i} l_{i} \leq k(d-c)+\max (0, l-c) .
$$

In addition, if $d<d^{\prime}<n$ then

$$
k(d-c)+\max (0, l-c) \leq k^{\prime}\left(d^{\prime}-c\right)+\max \left(0, l^{\prime}-c\right),
$$

where $n=k^{\prime} d^{\prime}+l^{\prime}$ with $0 \leq l^{\prime}<d^{\prime}$.

Proof. This becomes clear if one views $\lambda$ as a Young diagram of size $n$, and let $\mu=(d, \ldots, d, l)$, where $d$ is repeated $k$ times. Then $\mu$ can be obtained from $\lambda$ by moving down certain boxes of $\lambda$ (note that $n_{1} \geq d$ ). In addition, $\bar{\lambda}$ is a Young diagram of size $\sum_{i} l_{i}$, obtained from $\lambda$ by deleting the first $c$ columns. (Note that $\max \left(0, n_{1}-c\right) \geq \cdots \geq \max \left(0, n_{k}-c\right) \geq 0$.) The first assertion in the lemma means that the size of $\bar{\lambda}$ is not greater than the size of $\bar{\mu}=$ $(d-c, \ldots, d-c, \max (0, l-c))$, which is again obtained from $\bar{\lambda}$ by removing the first $c$ columns. It is clear that the number of boxes removed from $\mu$ to obtain $\bar{\mu}$ is at least the number of boxes removed from $\lambda$ to obtain $\bar{\lambda}$, whence the assertion follows. Moreover, this number of removed boxes does not increase if one uses $d^{\prime}$ instead of $d$ to form $\mu$, whence the second assertion follows. 
Lemma 4.3. Let $g \in \mathrm{GL}_{n}(2)$ be an element of order $|g|=2^{m+1}, m>0$. Let $d$ be the minimum polynomial degree of $g$. Suppose that $d<|g|$. Then

$$
j(g) \leq(n-l)\left(1-\frac{|g|}{2 d}\right)+\max \left(0, l-\frac{|g|}{2}\right),
$$

where $n \equiv l(\bmod d)$ and $0 \leq l<d$. If $d=|g|-1$ then $1-\frac{|g|}{2 d}=\frac{|g|-2}{2(|g|-1)}$.

Proof. We first show that the bound is attained. Write $n=k d+l$ with $k \geq 0$. Let $x=$ $\operatorname{diag}\left(J_{d}, \ldots, J_{d}, J_{l}\right)$, where $J_{d}$ occurs $k$ times. By Lemma 4.1, $j\left(J_{l}\right)=\max \left(0, l-\frac{|\bar{g}|}{2}\right)$ and $j\left(J_{d}\right)=$ $d-\frac{|g|}{2}$. So $j(g)=k\left(d-\frac{|g|}{2}\right)+\max \left(0, l-\frac{|g|}{2}\right)$ and $k=\frac{n-l}{d}$. Then $j(g)=\frac{n-l}{d}\left(d-\frac{|g|}{2}\right)+\max \left(0, l-\frac{|g|}{2}\right)$, as claimed.

The Jordan form of unipotent elements $g \in \mathrm{GL}_{n}(2)$ can be encoded by the Young diagrams, that is, $\left(n_{1}, \ldots, n_{k}\right)$ corresponds to $\operatorname{diag}\left(J_{n_{1}}, \ldots, J_{n_{k}}\right)$, where we assume $n_{1} \geq \cdots \geq n_{k}$, so $n_{1}$ is the minimum polynomial degree of $g$. Then the inequality in the lemma follows from Lemma 4.2 above. Indeed, if $g$ is as in the statement then $n_{1}=d>c=: \frac{|g|}{2}$.

We specify the result of Lemma 4.3 as follows to make it more convenient for use in the next section.

Lemma 4.4. Let $g \in \mathrm{GL}_{n}(2)$ be a 2-element.

(i) Suppose that $|g| \leq(q+1) / 2$.

$$
j(g) \leq \frac{n(q-3)}{2(q-1)}+\frac{q-7}{4} .
$$

(ii) Suppose that $|g|=q+1$. Then we have

$$
j(g) \leq \begin{cases}\frac{n(q-1)}{2 q} & \text { if } q \mid n ; \\ \frac{(n-1)(q-1)}{2 q} & \text { if } q \mid(n-1) ; \\ \frac{(n+1)(q-1)}{2 q}-1 & \text { if } q \mid(n+1) .\end{cases}
$$

Proof. (i) We have $|g| \leq(q+1) / 2$ and $l-\frac{|g|}{2} \leq \frac{|g|}{2}-2 \leq \frac{q+1}{4}-2=(q-7) / 4$. By Lemma 4.3 . we have $j(g) \leq n\left(1-\frac{|g|}{2 d}\right)+\frac{q-7}{4}$. Note that $1-\frac{|g|}{2 d} \leq 1-\frac{|g|}{2(|g|-1)}$ as $d \leq|g|-1$.

Furthermore, $|g| \leq(q+1) / 2$ implies

$$
1-\frac{|g|}{2(|g|-1)}=1-\frac{1}{2-(2 /|g|)} \leq 1-\frac{1}{2-(2 /(q+1)}=1-\frac{q+1}{2 q-2}=\frac{q-3}{2(q-1)} .
$$

So

$$
j(g) \leq \frac{n(q-3)}{2(q-1)}+\frac{q-7}{4} .
$$

(ii) Let $|g|=q+1=2^{m+1}$ and $d=|g|-1=q$.

(a) Let $q \mid n$. Then $j(g) \leq \frac{n(|g|-2)}{2(|g|-1)}=\frac{n(q-1)}{2 q}$.

(b) Let $q \mid(n-1)$. Then $j(g) \leq \frac{(n-1)(|g|-2)}{2(|g|-1)}=\frac{(n-1)(q-1)}{2 q}$.

(c) Let $q \mid(n+1)$. Then $l=d-1=|g|-2=q-1$ so

$$
j(g) \leq \frac{(n-q+1)(|g|-2)}{2(|g|-1)}+|g|-2-(|g| / 2)=\frac{(n+1)(q-1)}{2 q}-1 .
$$




\section{The CASE OF $\mathrm{SU}_{3}(q)$}

In this section we refine our results on minimal polynomials of elements of the group $\mathrm{SU}_{3}(q)$ in its cross-characteristic irreducible representations. The main result is Proposition 5.2 .

Lemma 5.1. Let $S=\mathrm{SU}_{3}(q) \leq G \leq H=U_{3}(q), 2 \nmid q$, and let $g \in G$ be a non-central semisimple 2-element, and let $|g|=2^{\alpha}$. Let $\phi \in \operatorname{IBr}_{\ell}(G)$ with $(\ell, q)=1$ and $\operatorname{dim} \phi>1$. Suppose that $\operatorname{deg} \phi(g)<o(g)$. Then one of the following holds:

(i) $\ell=2, g$ is not a pseudoreflection and $|g|$ divides $q+1$;

(ii) $q+1=2^{\alpha}, g$ is a pseudoreflection and $\operatorname{deg} \phi=o(g)-1$,

(iii) $q+1=2^{\alpha-1}, g^{2}$ is a pseudoreflection and $\operatorname{deg} \phi=o(g)-2$.

(iv) $|g|=q+1=4, g$ is not a pseudoreflection and $\operatorname{deg} \phi=3$;

In addition, in cases (ii), (iii) and (iv), $\Phi$ is a Weil representation of $G$.

Proof. Suppose first that $g$ is contained in a parabolic subgroup of $G$. Then, applying the main result of [11, Theorem 13.2] and [23, Theorem 3.2], we conclude that (ii) or (iii) holds.

Suppose that $g$ is not contained in any parabolic subgroup of $G$. Note that $g$ is contained in a maximal torus $T$ of $H$, and $|T| \in\left\{q^{3}+1,(q+1)\left(q^{2}-1\right),(q+1)^{3}\right\}$. The tori of order $(q+1)\left(q^{2}-1\right)$ lie in parabolic subgroups, and those of order $q^{3}+1$ contains no non-central 2-element. The torus of order $(q+1)^{3}$ is of exponent $q+1$, so $|g|$ divides $q+1$. If $\ell \neq 2$ or $|g|=q+1=4$ then the argument of the proof [55, Lemma 6.1] works (see page 653 there). So we have (i) and (iv).

We improve the conclusion in (i) of Lemma 5.1 as follows:

Proposition 5.2. Let $G=\mathrm{SU}_{3}(q)$, $q$ odd, and let $g \in G$ be a 2-element of order dividing $q+1$. Let $\phi$ be a non-trivial irreducible 2-modular representation of $G$. Then the minimum polynomial of $\phi(g)$ is of degree $|g|$, unless possibly $\operatorname{dim} \phi=q(q-1),|g|=q+1$ and $\operatorname{deg} \phi(g)=|g|-1$. If $\operatorname{deg} \phi(g)=|g|-1$ then the Jordan form of $\phi(g)$ consists of $q-1$ blocks of size $q$.

Before proving Proposition 5.2 , we deduce a consequence of it:

Corollary 5.3. Let $G=\mathrm{SU}_{3}(q)$, and let $g \in G$ be a p-element, $p \nmid q$. Let $o(g)$ be the order of $g$ modulo $Z(H)$ and let $\phi$ be a non-trivial irreducible $\ell$-modular representation of $G, \operatorname{dim} \phi>1$. Then $\operatorname{deg} \phi(g)=o(g)$, unless, possibly, $\phi$ is a Weil representation of $G$.

Proof. Note that $o(g)=|g|$ if $(p, q+1) \neq 3$, in particular, if $p \neq 3$. The result is contained in Proposition 5.2 if $p=2$ (as $\operatorname{dim} \phi=q^{2}-q$ implies $\phi$ to be Weil), and in [55, Lemma 6.1] for $p>2$.

The proof of Proposition 5.2 occupies the rest of this section. This is trivial if $|g|=2$ so we assume $|g| \geq 4$. Then $4 \mid(q+1)$. We start with some elementary observations.

Note that the involutions in $G=\mathrm{SU}_{3}(q)$ are conjugate. Denote by $z$ an involution from a parabolic subgroup $P$ of $G$. Let $U$ be the unipotent radical of $P$. We can write

$$
z=\left(\begin{array}{ccc}
-1 & 0 & 0 \\
0 & 1 & 0 \\
0 & 0 & -1
\end{array}\right) \quad U=\left\{u=\left(\begin{array}{ccc}
1 & a & c \\
0 & 1 & b \\
0 & 0 & 1
\end{array}\right)\right\}, \text { and } z u z^{-1}=\left(\begin{array}{ccc}
1 & -a & c \\
0 & 1 & -b \\
0 & 0 & 1
\end{array}\right)
$$

where $a \in \mathbb{F}_{q^{2}}^{\times}, c \in \mathbb{F}_{q}^{\times}$and $b=a^{q}$. So $|U|=q^{3},[z, Z(U)]=1$. If $\psi$ is an irreducible representation of $U$ of dimension not 1 then $\operatorname{dim} \phi=q$, the eigenvalues of $\phi(z)$ are $1,-1$, and their multiplicities are $(q-1) / 2$ and $(q+1) / 2$, with the multiplicity of -1 being even. As $4 \mid(q+1)$, we conclude that the multiplicity of 1 is $(q-1) / 2$. In fact, if $\psi$ is 2 -modular then the Jordan form of $\phi(z)$ has $(q-1) / 2$ blocks of size 2 . 
Lemma 5.4. Let $\phi$ be an irreducible 2-modular Brauer character of $G=\mathrm{SU}_{3}(q), 4 \mid(q+1)$. Let $z \in G$ be an involution. Denote by $j(\phi(z))$ the number of non-trivial Jordan blocks of $\phi(z)$. Then

$$
j(\phi(z)) \geq \frac{(q-1)\left(\phi(1)-\left(\left.\phi\right|_{Z(U)}, 1_{Z(U)}\right)\right)}{2 q}+\frac{\left(\left.\phi\right|_{Z(U)}, 1_{Z(U)}\right)-\left(\left.\phi\right|_{U}, 1_{U}\right)}{2} .
$$

Proof. Let $V$ be the underlying module of $\phi$. Then $\left.V\right|_{U}=V^{U} \oplus V_{1} \oplus V_{2}$, where $V_{1}+V^{U}=V^{Z(U)}$ and $V_{2}=[V, Z(U)]$. So $\operatorname{dim} V_{2}=\operatorname{dim} V-\operatorname{dim} V^{Z(U)}$. Then $V_{2}$ is the direct sum of irreducible $U$-modules non-trivial on $Z(U)$, so $V_{2}$ is the sum of $\frac{\operatorname{dim} V_{2}}{q}$ irreducible $U$-modules of dimension $q$. In addition, $V_{1}$ is the sum of non-trivial one-dimensional $U$-modules. As $C_{U}(z)=Z(U)$, it follows that no non-trivial linear character of $U$ is $z$-invariant; this implies the number of Jordan blocks of $z$ on $V_{1}$ to be equal to $\operatorname{dim} V_{1} / 2$. Therefore, the number in question equals

$$
j(\phi(z))=\frac{(q-1) \operatorname{dim} V_{2}}{2 q}+\frac{\operatorname{dim} V_{1}}{2} .
$$

As $\operatorname{dim} V^{U}=\left(\left.\phi\right|_{U}, 1_{U}\right), \operatorname{dim} V^{Z(U)}=\left(\left.\phi\right|_{Z(U)}, 1_{Z(U)}\right)$, and $\operatorname{dim} V_{1}=\operatorname{dim} V^{Z(U)}-\operatorname{dim} V^{U}$, the statement follows.

The terms of the formula in the lemma can be easily computed by using the character table of $G$ and the decomposition matrix of $G$ modulo 2. This is known to experts but the result is not explicitly written in literature. So we provide some detailed comments below.

There are three unipotent characters of $G$, of degree $1, q^{3}$ and $q^{2}-q$ [17]. The latter is irreducible modulo 2 [31, Proposition 9], and $\chi_{q^{3}}^{\circ}$ decomposes as $1_{G}+2 \chi_{q^{2}-q}^{\circ}+\phi_{0}$, where $\phi_{0}$ is irreducible of degree $q^{3}-2 q^{2}+2 q-1$ [30, Theorem 4.1]. This also shows that all these unipotent characters are in the principal block $B_{0}$. and they form a basic set for the union $\mathcal{E}_{2}(G, 1)$ of all $\mathcal{E}(G, s)$ with $s$ a 2-element by [19, Theorem A]. It follows that this union is precisely $B_{0}$, and $B_{0}$ contains 3 irreducible Brauer characters.

Now we consider $\chi \in \mathcal{E}_{2}(G, s)$, where $s \in G^{*}=\operatorname{PGU}_{3}(q)$ is semisimple of odd order $|s|>1$. Again by [19, Theorem A], the characters in $\mathcal{E}(G, s)$ form a basic set for $\mathcal{E}_{2}(G, s)$. The element $s$ belongs to a maximal torus $T *$ of $G^{*}$, of order $(q+1)^{2}, q^{2}-1$, or $q^{2}-q+1$.

Suppose first that $\left|T^{*}\right|=q^{2}-q+1$. Then $s$ is regular in $G^{*}$. If $|s|>3$ then $\mathcal{E}(G, s)$ consists of a unique character of degree $(q+1)\left(q^{2}-1\right)$. If $|s|=3$ then $3 \mid(q+1)$ and $\mathcal{E}(G, s)$ consists of three characters of degree $(q+1)\left(q^{2}-1\right) / 3$. These characters are all of 2 -defect 0 .

Suppose next that $\left|T^{*}\right|=q^{2}-1$ but $s$ does not belong to any torus of order $(q+1)^{2}$. Then $s$ is regular and $\left|C_{G^{*}}(s)\right|=q^{2}-1$. So $\mathcal{E}(G, s)$ consists of a unique character of degree $q^{3}+1$. As every Brauer character is an integral linear combination of ordinary characters in its block, we conclude that its degree is $q^{3}+1$ too, and it is liftable.

Suppose now that $\left|T^{*}\right|=(q+1)^{2}$. Then $|s|$ divides $q+1$ and one of the following holds:

(i) $s$ is regular. If $|s|>3$ then $\left|C_{G^{*}}(s)\right|=(q+1)^{2}$ and $\mathcal{E}(G, s)$ consists of a unique character of degree $(q-1)\left(q^{2}-q+1\right)$, hence, as above, the unique Brauer character in the block has the same degree. If $|s|=3$, then $\left|C_{G^{*}}(s)\right|=3(q+1)^{2}$, and $\mathcal{E}(G, s)$ consists of 3 characters $\chi_{1,2,3}$ of the same degree $(q-1)\left(q^{2}-q+1\right) / 3$. It follows that every Brauer character of $\mathcal{E}_{2}(G, s)$ is of degree divisible by $(q-1)\left(q^{2}-q+1\right) / 3$. Hence, each $\chi_{i}^{\circ}$ is irreducible, and, as $\chi_{1}, \chi_{2}, \chi_{3}$ form a basic set, we again see that each irreducible Brauer character is liftable.

(ii) $s$ is not regular. Then $C_{G^{*}}(s) \cong \mathrm{GU}_{2}(q)$, and $\mathcal{E}(G, s)$ consists of two characters, $\chi_{1}$ (a Weil character) of degree $q^{2}-q+1$, and $\chi_{2}$ of degree $q\left(q^{2}-q+1\right)$. It is well known that $\chi_{1}^{\circ}$ is irreducible, see e.g. [31, Proposition 9]. We can represent $s$ by a diagonal matrix $\operatorname{diag}(\alpha, \alpha, 1)$ in $\mathrm{GU}_{3}(q)$ with $\alpha \neq 1$ of odd order dividing $q+1$. Then the element $t \in G^{*}$ represented by $\operatorname{diag}(\alpha,-\alpha, 1)$ centralizes $s$ and has $s$ as its $2^{\prime}$-part, and $\mathcal{E}(G, t)$ consists of a unique character $\psi$ of degree $(q-1)\left(q^{2}-q+1\right)$. Note that $\psi(t)=2 q-1, \chi_{1}(t)=1-q$, and $\chi_{2}(t)=q$ for a transvection $t \in G$, see [17, Table 3.1]. Since $\psi^{\circ}$ is a linear combination of $\chi_{1}^{\circ}$ and $\chi_{2}^{\circ}$, it follows 
that $\chi_{2}^{\circ}=\psi^{\circ}+\chi_{1}^{\circ}$, and $\left\{\psi^{\circ}, \chi_{1}^{\circ}\right\}$ is a basic set for $\mathcal{E}_{2}(G, s)$. We claim that $\psi^{\circ}$ is irreducible. Indeed, $\psi^{\circ}=a \gamma+b \chi_{1}^{\circ}$ for some irreducible Brauer character $\gamma$ and some integers $a, b \geq 0$. Inspecting the multiplicity of $1_{U}$ and of any nontrivial linear character $\xi$ of $U$, using [17, Table 3.2], we obtain $\left(\left.\psi\right|_{U}, 1_{U}\right)=0=a\left(\left.\gamma\right|_{U}, 1_{U}\right)+b$ and $\left(\left.\psi\right|_{U}, \xi\right)=1=a\left(\left.\gamma\right|_{U}, \xi\right)$, whence $(a, b)=(1,0)$, i.e. $\psi^{\circ}=\gamma$, as claimed. Therefore, there are two irreducible Brauer characters in $\mathcal{E}_{2}(G, s)$, of degree $q^{2}-q+1$ and $(q-1)\left(q^{2}-q+1\right)$, and they both lift.

Thus, $\phi_{0}$ is the only non-liftable 2-modular irreducible Brauer character, and it is of degree $q^{3}-1-2\left(q^{2}-q\right)$.

Let $1 \neq u \in Z(U)$ and $v \in(U \backslash Z(U))$. Then $\phi(u), \phi(v)$ do not depend on the choice of $u, v$. Then

$\left(\left.\phi\right|_{Z(U)}, 1_{Z(U)}\right)=\frac{1}{q}(\phi(1)+(q-1) \phi(u))$ and $\left(\left.\phi\right|_{U}, 1_{U}\right)=\frac{1}{q^{3}}\left(\phi(1)+\phi(v)\left(q^{3}-q\right)+\phi(u)(q-1)\right)$.

For our purpose we could ignore irreducible representations of degree $(q+1)\left(q^{2}-1\right)$ as these are of 2-defect 0 , so the restrictions of them to the Sylow 2-subgroup of $G$ are the characters of projective modules.

Next, for every non-trivial irreducible 2-modular Brauer character $\phi$ of $G$ of non-zero defect we compute the multiplicity of $1_{Z(U)}$ in $\left.\phi\right|_{Z(U)}$ and the multiplicity of $1_{U}$ in $\left.\phi\right|_{U}$. This can be easily done by using the character table of $G$. The results are summarized in Table 1.

Table 1

\begin{tabular}{|c|c|c|c|c|c|c|}
\hline$\phi$ & $\phi(1)$ & $\phi(u)$ & $\left(\left.\phi\right|_{Z(U)}, 1_{Z(U)}\right)$ & $\phi(v)$ & $\left(\left.\phi\right|_{U}, 1_{U}\right)$ & $\left(\left.\phi\right|_{Z(U)}, 1_{Z(U)}\right)-\left(\left.\phi\right|_{U}, 1_{U}\right)$ \\
\hline$\phi_{1}$ & $q^{2}-q$ & $-q$ & 0 & 0 & 0 & 0 \\
\hline$\phi_{2}$ & $q^{2}-q+1$ & $-q+1$ & 1 & 1 & 0 & 1 \\
\hline$\phi_{4}$ & $(q-1)\left(q^{2}-q+1\right)$ & $2 q-1$ & $q^{2}-1$ & -1 & 0 & $q^{2}-1$ \\
\hline$\phi_{4}^{*}$ & $(q-1)\left(q^{2}-q+1\right) / 3$ & $(2 q-1) / 3$ & $\left(q^{2}-1\right) / 3$ & $x, y, y$ & 0 & $\left(q^{2}-1\right) / 3$ \\
\hline$\phi_{5}$ & $q^{3}+1$ & 1 & $q^{2}+1$ & 1 & 2 & $q^{2}-1$ \\
\hline$\phi_{7}$ & $q^{3}$ & 0 & $q^{2}$ & 0 & 1 & $q^{2}-1$ \\
\hline$\phi_{0}$ & $q^{3}-2 q^{2}+2 q-1$ & $2 q-1$ & $q^{2}-1$ & -1 & 0 & $q^{2}-1$ \\
\hline
\end{tabular}

In Table $1, x=(2 q-1) / 3, y=-(q+1) / 3$. Note that the character $\phi_{4}^{*}$ exists if and only if $3 \mid(q+1)$; in this case the set $U \backslash Z(U)$ is the union of three conjugacy classes, and $\phi_{4}^{*}(v)=y$ for $v$ in two of them, and $\phi_{4}^{*}(v)=x$ when $v$ lies in the remaining class. In fact, each irreducible representation of $U_{3}(q)$ of degree $(q-1)\left(q^{2}-q+1\right)$ restricts to $G$ as the sum of three irreducible representations of degree $(q-1)\left(q^{2}-q+1\right) / 3$. If $\phi_{4 a}, \phi_{4 b}, \phi_{4 c}$ are the characters of these representations of $G$ and $v_{1}, v_{2}, v_{3}$ are representatives of the three conjugacy classes in question then the corresponding fragment of the Brauer character table is

\begin{tabular}{|c|c|c|c|}
\hline & $v_{1}$ & $v_{2}$ & $v_{3}$ \\
\hline$\phi_{4 a}$ & $x$ & $y$ & $y$ \\
\hline$\phi_{4 b}$ & $y$ & $x$ & $y$ \\
\hline$\phi_{4 c}$ & $y$ & $y$ & $x$ \\
\hline
\end{tabular}

This is irrelevant for computation of $\left(\left.\phi_{4}^{*}\right|_{U}, 1_{U}\right)$. On the other hand, if $1_{U}$ does not occur as a constituent of $\left.\phi_{4}\right|_{U}$ then $1_{U}$ does not occur as a constituent of $\left.\phi_{4}^{*}\right|_{U}$.

Let

$$
f_{1}(n)=\frac{n(q-3)}{2(q-1)}+\frac{q-7}{4} \text { and } f_{2}(n)= \begin{cases}n(q-1) / 2 q & \text { if } n \equiv 0 \quad(\bmod q) \\ (n-1)(q-1) / 2 q & \text { if } n \equiv 1 \quad(\bmod q) \\ \frac{(n+1)(q-1)}{2 q}-1 & \text { if } n \equiv-1 \quad(\bmod q) .\end{cases}
$$

be the functions defined in Lemma 4.4(i), (ii), respectively. Then we have (where $n=\phi(1)$ ):

Table 2 


\begin{tabular}{|c|c|c|c|c|}
\hline$\phi$ & $n=\phi(1)$ & $j(\phi(z)) \geq$ & $f_{1}(n)$ & $f_{2}(n)$ \\
\hline$\phi_{1}$ & $q^{2}-q$ & $(q-1)^{2} / 2$ & $\left(2 q^{2}-5 q-7\right) / 4$ & $(q-1)^{2} / 2$ \\
\hline$\phi_{2}$ & $q^{2}-q+1$ & $(q-1)^{2} / 2$ & $\left(2 q^{3}-7 q^{2}+1\right) / 4(q-1)$ & \\
\hline$\phi_{4}$ & $(q-1)\left(q^{2}-q+1\right)$ & $(q-1)\left(q^{2}-2 q+3\right) / 2$ & $\left(2 q^{3}-8 q^{2}+9 q-13\right) / 4$ & $-1+\frac{(q-1)\left(q^{2}-2 q+2\right)}{2}$ \\
\hline$\phi_{4}^{*}$ & $(q-1)\left(q^{2}-q+1\right) / 3$ & $(q-1)\left(q^{2}-2 q+3\right) / 6$ & $\left.q^{3}-7 q^{2}+5 q-12\right) / 6$ & \\
\hline$\phi_{5}$ & $q^{3}+1$ & $(q-1)\left(q^{2}+1\right) / 2$ & $\frac{2 q^{4}-6 q^{3}+q^{2}-6 q+1}{4(q-1)}$ & $q^{2}(q-1) / 2$ \\
\hline$\phi_{0}$ & $(q-1)\left(q^{2}-q+1\right)$ & $(q-1)\left(q^{2}-2 q+3\right) / 2$ & $\left(2 q^{3}-8 q^{2}+9 q-13\right) / 4$ & $-1+\frac{(q-1)\left(q^{2}-2 q+2\right)}{2}$ \\
\hline
\end{tabular}

In Table 2 we have left blank certain positions in the fifth column as this column is created under assumption that $|g|=q+1$. Recall that if $q+1$ is a 2-power then there are no irreducible 2 -modular representations of degree $q^{2}-q+1$ and $(q-1)\left(q^{2}+q+1\right) / 3$. Note that the third column gives the lower bound for $j(\phi(z))$ from Lemma 5.4 obtained using Table 1 .

Proof of Proposition 5.2. Let $d=\operatorname{deg} \phi(g)$. Suppose the contrary, that $d<|g|$. Let $n=\operatorname{dim} \phi$. Suppose first that $|g| \neq q+1$. Then $|g| \leq(q+1) / 2$. Let $z$ be the involution in $\langle g\rangle$, so $j(\phi(g))$ is the number of non-trivial blocks in the Jordan form of $\phi(z)$. By Lemma 5.4, $j(\phi(g)) \geq t(n)$, where $t(n)$ is given in the 3 -rd column of Table 2 .

By Lemma 4.4 $d<|g|$ implies $j(\phi(g)) \leq f_{1}(n)$, so $t(n) \leq f_{1}(g)$. One easily observes that this is false, whence a contradiction.

Let $|g|=q+1$. Inspecting the second column of Table 2 , one observes that $\phi_{i}(1)(\bmod q) \in$ $\{1,0,-1\}$ for $i=1,4,5,6$. So we can use Lemma 4.4(ii) to build the upper bound for $j(\phi(g))$, which is written in the 5 -th column there. As above, we compare the entries of the 3rd and 5 th columns of Table 2, to observe that these are not compatible for $i=4,5,0$. (There is no contradiction for $i=1$.)

Let $n=\phi(1)=q^{2}-q$. Then $\mathrm{GL}_{n}(2)$ contains the matrix $J:=\left(J_{q}, \ldots, J_{q}\right)$, where $J_{q}$ is repeated $q-1$ times. Then $j(J)=(q-1)^{2}$ by Lemma 4.2. Let $\left(J_{n_{1}}, \ldots, J_{n_{k}}\right)$ be the Jordan form of $\phi(g)$, where $n_{1} \geq \cdots \geq n_{k}$. If this is not $J$ then, by Lemma $4.2, j(\phi(g))<(q-1)^{2}$, which is a contradiction (by Table 2).

\section{Groups OF BN-PAIR RANK 1}

\subsection{Groups ${ }^{2} B_{2}\left(2^{2 m+1}\right)$ and ${ }^{2} G_{2}\left(3^{2 m+1}\right)$.}

Lemma 6.1. Let $G={ }^{2} B_{2}\left(2^{2 m+1}\right), m>0$, be a Suzuki group. For $p>2$ let $g \in G$ be a p-element. Let $1_{G} \neq \phi \in \operatorname{Irr}_{\ell} G$ and $\ell \neq 2$. Then $\operatorname{deg} \phi(g)=|g|$.

Proof. Note that Sylow $p$-subgroups of $G$ are cyclic. If $\ell=0$ or $\ell=p$, the result follows from [60], see also [59, 2.8] for $\ell=0$. Suppose $\ell \neq 0, p$. Let $\beta$ be the Brauer character of $\phi$. If $\beta$ is liftable, the result follows from [60]. The decomposition numbers of $G$ have been determined by Burkhardt [3. Inspection of them in [3] shows that $\phi$ either liftable or St $(x)-1=\phi(x)$ for every $l^{\prime}$-element $x \in G$. As $\operatorname{St}(x) \in\{ \pm 1\}$ and $\operatorname{St}(1)=2^{2(2 m+1)}$, the result easily follows from Lemma 2.1.

Lemma 6.2. Let $G={ }^{2} G_{2}(q), q=3^{2 m+1}, m>0$. Let $g \in G$ be a p-element for some prime $p \neq 3$ dividing $|G|$. Let $\ell \neq 3, \phi \in \operatorname{Irr}_{\ell} G, \operatorname{dim} \phi>1$. Then $\operatorname{deg} \phi(g)=|g|$.

Proof. The lemma is trivial for $p=2$, as every 2-element of $G$ is an involution.

Let $p>2$. Then Sylow $p$-subgroups of $G$ are cyclic. Let $\beta$ be the Brauer character of $\phi$. If $\ell \in\{0, p\}$ or $\beta$ is liftable, the result follows from [60]. Suppose otherwise.

Let $\ell=2$. By [38, p.104], $\beta$ lies in the principal block and $\beta \in\left\{\beta_{1}, \beta_{2}\right\}$, where $\beta_{2}=\xi_{2}-1_{G}$ and $\beta_{3}=\mathrm{St}^{\circ}+1_{G}-2 \xi_{2}^{\circ}-\xi_{6}^{\circ}-\xi_{8}^{\circ}$ in notation of [38].

Let $\ell>2$. Then the Sylow $\ell$-subgroups of $G$ are cyclic. The Brauer tree for $G$ for every $\ell>2$ is determined by Hiss [29]. Inspection in [29] shows that $\beta$ either liftable or $\ell \mid\left(3^{2 m+1}+1\right)$ and $\beta_{1}=\mathrm{St}^{\circ}-1_{G}-\xi_{5}^{\circ}-\xi_{7}^{\circ}$, or $\ell \mid\left(3^{2 m+1}-3^{m+1}+1\right)$ and $\beta_{4}=\mathrm{St}^{\circ}-1_{G}$, in notation of [29, 58]. 
It suffices to show that the restriction $\left.\beta_{i}\right|_{C}-\rho_{C}^{\text {reg }}$ is either 0 or a proper character of $C$ for every maximal cyclic $p$-subgroup $C$ of $G$. There are 4 maximal tori of $G$; these are cyclic groups of order $\left|T_{1}\right|=q-1,\left|T_{2}\right|=q+1,\left|T_{3}\right|=q+\sqrt{3 q}+1$ and $\left|T_{4}\right|=q-\sqrt{3 q}+1$. We write $C=C_{i}$ if $|C|$ divides $\left|T_{i}\right|$.

Inspection of the character table of $G$ in $\left[58\right.$ ] shows that every character $\xi_{j}(j=2,5,6,7,8)$ as well as St is constant at $C \backslash\{1\}$. Therefore, the result follows by applying Lemma 2.1 to the values of these characters (given in [58]).

For reader's convenience in the following table we give the values of the characters involved at $1 \neq t \in C_{i}$ for $i=1,2,3,4$.

\begin{tabular}{|c|c|c|c|c|c|c|c|c|c|c|c|}
\hline & $\mathrm{St}$ & $\xi_{2}$ & $\xi_{4}$ & $\xi_{5}$ & $\xi_{6}$ & $\xi_{7}$ & $\xi_{8}$ & $\beta_{1}$ & $\beta_{2}$ & $\beta_{3}$ & $\beta_{4}$ \\
\hline$C_{1}$ & 1 & 1 & 1 & 0 & 0 & 0 & 0 & 0 & 0 & 0 & 0 \\
\hline$C_{2}$ & -1 & 3 & -3 & 1 & -1 & 1 & -1 & -4 & 2 & -4 & -2 \\
\hline$C_{3}$ & -1 & 0 & 0 & -1 & 0 & -1 & 0 & 0 & -1 & 0 & -2 \\
\hline$C_{4}$ & -1 & 0 & 0 & 0 & 1 & 0 & 1 & -2 & -1 & -2 & -2 \\
\hline
\end{tabular}

Note that $\xi_{4}(1)=q^{2}\left(q^{4}-q^{2}+1\right), \xi_{2}(1)=q^{2}-q+1$ and $\xi_{6}(1)=\xi_{8}(1)=\left(q^{2}-1\right) q\left(q^{2}+1-\right.$ $q \sqrt{3}) / 2 \sqrt{3}$. So $\beta_{2}(1)=\xi_{4}(1)-\xi_{2}(1)-\xi_{6}(1)-\xi_{8}(1)=\left(\left(q^{6}-q^{2}-q+1\right)+q\left(q^{2}-1\right)\left(q^{2}+1-\right.\right.$ $q \sqrt{(3)}) / \sqrt{3})$.

\section{The CASE OF $G_{2}(q)$}

In this section we prove Theorem 1.1 for $G=G_{2}(q), q>2$, and $\ell \neq 2$. The case of $G=G_{2}(2)$ is considered in Lemma 7.13 at the end of this section. (Also see Lemma 7.14 for group $2 \cdot G_{2}(4)$.)

Theorem 7.1. Theorem 1.1 is true for $G=G_{2}(q), q=r^{a}>2$.

Lemma 7.2. Let $G=G_{2}(q)$ and $q \geq 3$. Then every semisimple element of $G$ is contained in a subgroup isomorphic to $\mathrm{SL}_{3}(q)$ or $\mathrm{SU}_{3}(q)$.

Proof. Every semisimple element is contained in a maximal torus of $G$. Also, $G$ has 6 conjugacy classes of maximal tori, whose orders are $q^{2}-q+1, q^{2}+q+1,(q-1)^{2},(q+1)^{2}$ and two classes of order $q^{2}-1$. In addition, $G$ contains subgroups $L^{+} \cong \mathrm{SL}_{3}(q)$ and $L^{-} \cong \mathrm{SU}_{3}(q)$, whose maximal tori are maximal in $G$. If $3 \mid q$, then the statement follows from fusion of conjugacy classes of certain subgroups $L^{+} \cong \mathrm{SL}_{3}(q)$ and $L^{-} \cong \mathrm{SU}_{3}(q)$ in $G$ as given in [14]: every semisimple class of $G$ intersects $L^{+}$or $L^{-}$.

Assume now that $3 \nmid q$ and choose $\varepsilon \in\{+,-\}$ such that $3 \mid(q-\varepsilon)$. It suffices to show that maximal tori $T^{+}<L^{+}$and $T^{-}<L^{-}$(both cyclic groups of order $q^{2}-1$ ) are not conjugate in $G$. Assume the contrary: $T^{+}$and $T^{-}$are conjugate in $G$. It is shown in [5] and [15] that $G$ has two conjugacy classes of elements of order 3 , with representatives $u$, $v$, where $C_{G}(u) \cong \mathrm{SL}_{3}^{\varepsilon}(q)=L^{\varepsilon}$, and $C_{G}(v) \cong \mathrm{GL}_{2}^{\varepsilon}(q)$. Then $T^{\varepsilon}$ contains $Z\left(L^{\varepsilon}\right)$, and so $u \in T^{\varepsilon}$. As $T^{-\varepsilon}$ is conjugate to $T^{\varepsilon}$, we may assume that $u \in T^{-\varepsilon}<L^{-\varepsilon}$.

Consider the case with $\varepsilon=-$ and $\chi \in \operatorname{Irr}(G)$ of degree $q^{3}-1$. Then $\chi(u)=-q(q-1)$, see [5] and [15. On the other hand, if $\theta \in \operatorname{Irr} \operatorname{SL}_{3}(q)$ and $\theta(1) \leq q^{3}-1$, then $\theta(g) \geq 0$, or $\theta(1)=q^{3}-1$ and $\theta(g)=-2$ for any element $g$ of order 3 . Restricting $\chi$ to $L^{+}$, we arrive at a contradiction.

Now assume that $\varepsilon=+$, and take $\chi \in \operatorname{Irr}(G)$ of degree $q^{3}+1$. Then $\chi(u)=q(q+1)$, see [5] and [15]. We use the notation of [17], and decompose

$\left.\chi\right|_{L^{-}}=a \chi_{1}+b \chi_{q^{2}-q}+c \chi_{q^{3}}+\sum_{i=1}^{q}\left(d_{i} \chi_{q^{2}-q+1}^{(i)}+e_{i} \chi_{q\left(q^{2}-q+1\right)}^{(i)}\right)+\sum_{i, j} f_{i j} \chi_{(q-1)\left(q^{2}-q+1\right)}^{(i, j)}+\sum_{j} g_{j} \chi_{q^{3}+1}^{(j)}$,

where $a, b, c, \ldots$ are non-negative integers. First, if some $g_{j} \geq 1$, then $\left.\chi\right|_{L^{-}}=\chi_{q^{3}+1}^{(j)}$, and hence $|\chi(u)| \leq 2$, a contradiction. So $g_{j}=0$ for all $j$. Likewise, if $c \geq 1$, then $c=1,\left.\chi\right|_{L^{-}}=\chi_{1}+\chi_{q^{3}}$, 
yielding $\chi(u)=2$, again a contradiction. Now, evaluating at an element of order $q^{2}-q+1$, we get $0=a-b$, i.e. $b=a$. Comparing the degrees, we obtain

$$
q^{3}+1=\left(q^{2}-q+1\right)\left(a+\sum_{i} d_{i}+\sum_{i} q e_{i}\right)
$$

whence $a+\sum_{i} d_{i}+\sum_{i} q e_{i} \leq q+1$. Now, evaluating at $u$, we get

$$
q(q+1)=\chi(u)=2 a+\sum_{i} d_{i}+\sum_{i} e_{i} \leq q+1+a \leq 2(q+1),
$$

again a contradiction.

If $r \neq 3$ then the subgroups of $G$ isomorphic to $H_{i}$ for $i=1$ or 2 are conjugate, as follows from the classification of maximal subgroups of $G$ obtained in [7] and [35]. The case with $r=3$ has features which force us to consider this separately (Lemma 7.10).

Lemma 7.3. [62, Lemma 4.10] Let $p, q>2, p \nmid q$ and let $g \in G \cong G_{2}(q)$ be a p-element contained in a proper parabolic subgroup of $G$. Let $1_{G} \neq \theta \in \operatorname{Irr}_{\ell} G$. Then $\operatorname{deg} \theta(g)=|g|$.

Remark 7.4. In [62, Lemma 4.10] it is assumed that $\ell \neq p$, however, this is nowhere used in the prove of [62, Lemma 4.10]; so the claim is true for $\ell=p$ as well.

Lemma 7.5. Let $G=G_{2}(q), q>2$, and let $\theta \in \operatorname{Irr}_{F} G$ with $\operatorname{dim} \theta>1$. Let $g \in G$ be $a$ semisimple p-element. Suppose that $g \in H=\mathrm{SL}_{3}(q)$. Then $\operatorname{deg} \theta(g)=|g|$.

Proof. Suppose the contrary. Let $\phi$ be a non-trivial irreducible constituent of $\left.\theta\right|_{H}$. If $g$ is contained in a parabolic subgroup of $H$, then the result follows from Corollary 2.4, unless $g \in Z(H)$, so $|g|=3$. Then then it normalizes a nontrivial $r$-subgroup, whence $g$ lies in a parabolic subgroup of $G$, and the result follows from Lemma 7.3. So we assume that $g$ is not in a parabolic subgroup of $H$. Then $p>3$ and, by Lemma 2.6, $|g|=q^{2}+q+1$. Then Sylow $p$-subgroups of $G$ are cyclic. If $\ell=p$ or $\ell=0$ then the result in this case follows from [60]. Suppose that $\ell \neq 0, p$. Then, again by Lemma 2.6, $\operatorname{deg} \phi(g)=\operatorname{dim} \phi=q^{2}+q$, and 1 is not an eigenvalue of $\phi(g)$. If $1_{H}$ is a constituent of $\left.\theta\right|_{H}$ then $\operatorname{deg} \theta(g)=|g|$. Otherwise, $\operatorname{dim} \theta$ is a multiple of $q^{2}+q$. Then $\theta$ is liftable (this follows by inspection of Brauer character degrees of $G$ available in [49, 50] for $\left.\ell \mid\left(q^{2}+q+1\right)\right)$, and we are back to the complex case.

In view of Lemma 7.5 to prove Theorem 7.1 we can assume that $g \in H \cong \mathrm{SU}_{3}(q)$. Moreover, by [55, Lemma 6.1], if $p>2$ then either $|g|=q+1$ or $|g|=q^{2}-q+1$. In the former case $q$ is even. Let $p=2$. Then, by Lemma 5.1 and Proposition 5.2, either $|g|=q+1$ or $2(q+1)$.

For $H=\mathrm{SU}_{3}(q)$ the arguments in the proof of Lemma 7.5 do not work. Indeed, if $p \mid(q+1)$ then we cannot use [60] for $p=\ell$ as Sylow $p$-subgroups of $G$ are not cyclic. So we turn to another method. We show that the restriction to $H$ of every non-trivial irreducible representation of $G$ contains a non-trivial irreducible constituent which is not Weil. This will imply Theorem 7.1 in view of Proposition 5.2 for $p=2$ and [55, Lemma 6.1]. In addition, to handle the case of ${ }^{3} D_{4}(q)$ we need a similar result for $H=\mathrm{SL}_{3}(q)$ and $\ell=q^{2}+q+1$. In fact, for our use it suffices to consider the case where $Z(H)=1$. To deal with $\ell \mid\left(q^{4}+q^{2}+1\right)$ we first prove the result for $\ell=0$ and next use the decomposition numbers to deal with $\ell$-Brauer characters. In our reasoning below a certain role is played by the Gelfand-Graev representation of $H$. This is the induced representation $\lambda^{G}$, where $\lambda$ is a so-called non-degenerate linear character of the maximal unipotent subgroup of $G$.

Lemma 7.6. Let $G=G_{2}(q), q>2$, and $H \cong \mathrm{SL}_{3}^{\varepsilon}(q)<G,(3, q-\varepsilon)=1$. Let $1_{G} \neq \tau \in \operatorname{Irr}(G)$ (so $\ell=0$ here). Then $\left.\tau\right|_{H}$ contains a non-trivial irreducible constituent which is not Weil.

Proof. Let $U$ be a maximal unipotent subgroup of $H$ and let $\Gamma=\Gamma^{\varepsilon}$ be the Gelfand-Graev character of $H$. Recall that $Z(H)=1$ implies that the Gelfand-Graev representation of $H$ is unique (this follows from [10, 14.28 and 14.29]). It is well known that neither $1_{H}$ nor Weil are constituents of $\Gamma$. (Indeed, if $r \mid q$ is a prime then the degrees of any irreducible constituent of 
$\Gamma$ is $\left|C_{H}(s)\right|_{r} \cdot\left(|H| /\left|C_{H}(s)\right|\right)_{r^{\prime}}$ for some semisimple element $s \in H$, see [4, Theorem 8.4.9]. One observes that $1, q^{2}-\varepsilon q, q^{2}-\varepsilon q+1$ are not of these form.) Therefore, it suffices to show that

$$
\left(\left.\tau\right|_{H}, \Gamma\right)>0
$$

for every non-trivial irreducible character $\tau$ of $G$. As the character table of $G$ is known, this can be easily checked. Indeed, observe that $\Gamma(x)=0$ if $x \in H$ is not unipotent. In addition, $\Gamma(1)=|H| /|U|$ and $\Gamma(u)=1$ if $u \in H$ is regular unipotent [10, 14.45]. To compute $\Gamma(t)$ for $t \in H$ a transvection, one can use the inner product $\left(\Gamma, 1_{H}\right)=0$. The conjugacy classes $u^{H}, t^{H}$ of $u, t$, are of size $|H| / q^{2}=q|H| /|U|$ and $|H| /\left|C_{H}(t)\right|=|H| /|U|(q-\varepsilon)$, respectively. So we have

$$
\Gamma(t) \cdot\left|C_{H}(t)=\right| \Gamma(t)|H| /|U|(q-\varepsilon)=\Gamma(u) q|H| /|U|-|H| /|U|,
$$

whence $\Gamma(t) /(q-\varepsilon)=q-1$ and $\Gamma(t)=(q-\varepsilon)(q-1)$. Therefore,

$$
|H|\left(\left.\tau\right|_{H}, \Gamma\right)=\tau(1) \frac{|H|}{|U|}+\tau(t) \Gamma(t)\left|C_{H}(t)\right|+\tau(u)\left|C_{H}(u)\right|=\frac{|H|}{|U|}(\tau(1)+\tau(t)(q-1)+q \tau(u)),
$$

so $\left(\left.\tau\right|_{H}, \Gamma\right)>0$ if and only if $\tau(1)+\tau(t)(q-1)+q \tau(u)>0$. Inspection of the character table of $G$ in [5] (for $(q, 6)=1$ ), [14] (for $3 \mid q$ ) and [15] (for 2|q) yields the result.

Lemma 7.7. Let $H=\mathrm{SL}_{3}^{\varepsilon}(q)$ and let $\tau \in \operatorname{Irr} H$ with $\tau(1)>q^{2}+\varepsilon q+1$. Then $\tau^{\circ}$ has an irreducible constituent of degree greater than $q^{2}+\varepsilon q+1$.

Proof. The result can be easily deduced from the comments in [55, Examples 3.1 and 3.2].

Lemma 7.8. Let $G, H$ be as in Lemma 7.6. Let $1_{G} \neq \phi \in \operatorname{Irr}_{\ell} G$. Suppose that $\phi+m \cdot 1_{G}$ is liftable for some integer $m \geq 0$. Then $\left.\phi\right|_{H}$ contains a non-trivial irreducible constituent which is not Weil.

Proof. Let $\tau \in \operatorname{Irr}(G)$ be a character of $G$ such that $\tau^{\circ}=\phi+m \cdot 1_{G}$. Then the non-trivial irreducible Brauer characters of $H$ occurring in $\left.\phi\right|_{H}$ are the same as those in $\left(\left.\tau\right|_{H}\right)^{\circ}$. By Lemma 7.6. there is an irreducible constituent $\nu$ in $\left.\tau\right|_{H}$ such that $\nu(1)>q^{2}+\varepsilon q+1$. By Lemma 7.7, $\nu^{\circ}$ has an irreducible constituent of degree greater than $q^{2}+\varepsilon q+1$. Whence the lemma.

Lemma 7.9. Let $G, H$ be as in Lemma 7.6 and $1_{G} \neq \phi \in \operatorname{Irr}_{\ell} G$, where $\ell \mid\left(q^{4}+q^{2}+1\right)$. Then $\left.\phi\right|_{H}$ contains a non-trivial irreducible constituent which is not Weil.

Proof. (i) Recall that $\Gamma=\lambda^{H}$ for the Gelfand-Graev character $\Gamma$ of $H$, where $\lambda$ is a linear character of $U$. Therefore, $\Gamma^{\circ}=\left(\lambda^{\circ}\right)^{H}$, so $\Gamma^{\circ}$ is the Brauer character of a projective $F H$ module. In particular, if $\alpha$ is any class function on $H$, then the inner product $\left(\alpha^{\circ}, \Gamma^{\circ}\right)^{\prime}$ over $\ell^{\prime}$-elements of $H$ is equal to the usual inner product $(\alpha, \Gamma)$ over all elements in $H$. Also, as $\Gamma$ is multiplicity free, it follows that $\Gamma^{\circ}$ is a sum of Brauer characters of indecomposable projective modules $P_{\psi_{i}}$ of $H$, each of them occurs with multiplicity 1 . Therefore, $\left(\left.\phi\right|_{H}, \Gamma^{\circ}\right)^{\prime}$ equals the sum of multiplicities of the irreducible constituents $\nu$ of $\left.\phi\right|_{H}$ such that $\left(\nu, \Gamma^{\circ}\right)^{\prime}>0$.

Note that $\left(1_{H}, \Gamma^{\circ}\right)^{\prime}=\left(1_{H}, \Gamma\right)=0$ and hence $\nu \neq 1_{H}^{\circ}$. Let $\mu$ be a irreducible Weil character of $H$; in particular, $\left(\mu, \Gamma^{\circ}\right)^{\prime}=(\mu, \Gamma)=0$ as mentioned above. It is well known that $\mu^{\circ}=\mu^{\prime}+a \cdot 1_{H}$, for some $\mu^{\prime} \in \operatorname{Irr}_{\ell} H$ and $a \geq 0$. Therefore, $\left(\mu^{\prime}, \Gamma^{\circ}\right)^{\prime}=\left(\mu, \Gamma^{\circ}\right)^{\prime}=0$, so $\mu^{\prime} \neq \nu$. It follows that $\left.\phi\right|_{H}$ has an irreducible constituent that is neither trivial nor Weil if and only if $\left(\left.\phi\right|_{H}, \Gamma^{\circ}\right)^{\prime}>0$.

(ii) We will now prove that $\left(\left.\phi\right|_{H}, \Gamma^{\circ}\right)^{\prime}>0$. Denote by $\operatorname{Irr}_{\ell}^{*} G$ the set of characters $\phi \in \operatorname{Irr}_{\ell} G$ that are not of the form $\tau^{\circ}-a \cdot 1_{G}$ for some $\tau \in \operatorname{Irr}(G)$ and $a \geq 0$. Suppose first that $\phi \notin \operatorname{Irr}_{\ell}^{*} G$, so that $\phi=\tau^{\circ}-a \cdot 1_{G}$ for some $\tau \in \operatorname{Irr}(G)$ and $a \geq 0$. Then $\left(\phi, \Gamma^{\circ}\right)^{\prime}=\left(\left(\left.\tau\right|_{H}\right)^{\circ}, \Gamma^{\circ}\right)^{\prime}=\left(\left.\tau\right|_{H}, \Gamma\right)$, and (7.1) implies that $\left(\left.\tau\right|_{H}, \Gamma\right)>0$.

Suppose that $\phi \in \operatorname{Irr}_{\ell}^{*} G$. As in Lemma 7.6, we observe that $\left(\phi, \Gamma^{\circ}\right)^{\prime}>0$ if and only if

$$
\phi(1)+(q-1) \phi(t)+q \phi(u)>0 .
$$

To verify the inequality 7.2 we need the character values of $\phi$ at $u, t$, where $t \in H$ is a transvection and $u$ is a regular unipotent element of $H$. These can be computed from the 
decomposition numbers of $G$ modulo $\ell$, which are available in Shamash [49, 50] for $3 \neq \ell \mid\left(q^{2}+\right.$ $\varepsilon q+1)$ and [29] for $\ell \mid\left(q^{2}-1\right)$.

Suppose first that $3 \neq \ell \mid\left(q^{2}+\varepsilon q+1\right)$.

In notation of [29] the characters in $\operatorname{Irr}_{\ell}^{*} G$ that are in the principal block are $\left(X_{3}-X_{18}\right)^{\circ}$ if $\varepsilon=1$, and $\left(X_{12}-X_{16}+1_{G}\right)^{\circ}$ if $\varepsilon=-1$. From this one easily checks $(7.2)$.

Suppose that $\phi$ is not in the principal block. Then either $\phi$ is liftable or $\ell \mid\left(q^{2}-\varepsilon q+1\right)$ and $3 \mid(q-\varepsilon)$, in each case there is a single non-liftable character. If $\varepsilon=1$ then the non-liftable character are $\left(X_{33}-X_{32}\right)^{\circ}$ and if $\varepsilon=-1$ then this is $\left(X_{31}-X_{32}\right)^{\circ}$, see [29, p.190]. As above, one easily checks the above inequality $(7.2)$.

For $(q, 6) \neq 1$ and $\ell \mid\left(q^{2}+\varepsilon q+1\right)$ the decomposition numbers are determined by Shamash [50]. The decomposition numbers for the characters in the principal block and, for $2 \mid q$, in the other blocks containing non-liftable characters are the same as for $q$ with $(q, 6)=1$. If $3 \mid q$ then all non-liftable characters are in the principal block. The character tables of $G_{2}(q)$ with $(q, 6)>0$ can be found in [14] for $3 \mid q$ and [15] for 2|q. Inspection of [15] shows that $X_{i}(1)$ and $X_{i}(t)$ for non-liftable characters $X_{i}$ are the same polynomials in $q$ as for $q$ with $(q, 6)=1$. In addition, the absolute value of $X_{i}(u)$ is small enough to satisfy the inequality (7.2).

We are left with the cases where $\ell \mid\left(q^{2}-1\right)$. As the character table of $G_{2}(q)$ with $3 \mid q$ differs from that for $q$ with $(q, 3)=1$, we consider this case separately.

Lemma 7.10. Theorem 1.1 is true for $q=3^{m}$.

Proof. By Lemma 7.5 and the comments following it, we may assume that $p=2$ and $q+1$ is a 2-power. We assume $|g|>2$ as the case $|g|=2$ is trivial. Observe that $|q+1|_{2}=4$ if $\mathrm{m}$ is odd and $|q+1|_{2}=2$ otherwise. Therefore $q=3$. If $\ell \neq 2$ then the result follows by the Brauer character table of $G_{2}(3)$ [33].

Let $\ell=2$. Let $H \cong \mathrm{SU}_{3}(3)<G$. We show that $\left.\phi\right|_{H}$ has a non-trivial irreducible constituent which is not Weil. As above, it suffices to check that $(\phi, \Gamma)>0$, where $\Gamma$ is the Gelfand-Graev character of $H$. This holds if $\phi(1)+(q-1) \phi(t)+q \phi(u)>0$. This can be easily checked using the Brauer character table of $G$ for $\ell=2$ in 33 .

From now on, until the end of this subsection we assume $r \neq 3$. To complete the proof of Theorem 7.1 it suffices to show that $\left.\phi\right|_{H}$ has a non-trivial constituent which is not Weil (here $\left.\phi \in \operatorname{Irr}_{\ell} G, 3 \neq \ell \mid\left(q^{2}-1\right)\right)$. We can do this by the method used in the proof of Lemma 7.9. however, there is a more conceptual approach to do this.

Lemma 7.11. Let $G=G_{2}(q), q>2,3 \nmid q$, and let $P$ be a long-root parabolic subgroup of $G$. Let $U$ be the unipotent radical of $P, V$ be a non-trivial irreducible $F G$-module and $V_{0}=C_{V}(Z(U))$. Then $U / Z(U)$ acts on $V_{0}$ faithfully.

Proof. Assume the contrary: $U / Z(U)$ acts non-faithfully on $V_{0}$. It is well known that for $q>2$, $(q, 3)=1$ the group $U / Z(U)$ has no non-trivial proper $P$-invariant subgroup. (See [1] or [42, Theorem 17.6] for $(q, 6)=1$ and [37] for $q$ even; note that this fails for the excluded case $q=2)$. As $V_{0}$ is a $F P$-module, it follows that $U$ acts trivially on $V_{0}$, i.e. $V_{0}=C_{V}(U)$ and thus $V=[V, Z(U)] \oplus V_{0}$.

It is also well known that $U$ is generated by the root subgroups $U_{\beta}, U_{\alpha+\beta}, U_{2 \alpha+\beta}, U_{3 \alpha+\beta}$, $U_{3 \alpha+2 \beta}$ and $[U, U]=U_{3 \alpha+2 \beta}=\left[U_{\beta}, U_{3 \alpha+\beta}\right]=Z(U)$. Moreover, for every $1 \neq y \in(U \backslash Z(U))$ we have $[U, y]=Z(U)$. It follows that the character of every irreducible representation of $U$ non-trivial on $Z(U)$ vanishes on $y$.

Note that there exists some $y \in(U \backslash Z(U))$ so that the $G$-conjugacy class of $y$ meets $Z(U)$, say at a (long-root) element $x$; indeed, both $U_{3 \alpha+\beta}, U_{3 \alpha+2 \beta}$ are long root subgroups of $G$, and hence $G$-conjugate. Let $\varphi_{0}$ and $\varphi_{1}$ denote the Brauer characters of $V_{0}$ and $[V, Z(U)]$. Then $\varphi_{0}(x)=\varphi_{0}(y)=\operatorname{dim} V_{0}$, and $\varphi_{1}(y)=0$. On the other hand, all elements of $Z(U)$ are conjugate in $G$, so all non-trivial irreducible representations of $Z(U)$ occur in $\left.V_{2}\right|_{Z(U)}$ with the 
same multiplicity, whence $\varphi_{1}(x)=-\varphi_{1}(1) /(q-1)<0$. Thus $\left(\varphi_{0}+\varphi_{1}\right)(x) \neq\left(\varphi_{0}+\varphi_{1}\right)(y)$, a contradiction.

Lemma 7.12. Let $G=G_{2}(q), q>2,3 \nmid q$, and let $V$ be a non-trivial irreducible $F G$-module. Let $H \cong \mathrm{SU}_{3}(q)<G$. Then the restriction of $V$ to $H$ has a non-trivial composition factor which is not an irreducible Weil module.

Proof. Recall that $G$ has a single conjugacy class of subgroups isomorphic to $\mathrm{SU}_{3}(q)$ if $3 \nmid q$, see [35] and [7].

We use notation of Lemma 7.11. Let $r$ be a prime dividing $q$. Let $P$ be a parabolic subgroup specified in Lemma 7.11 and $U$ the unipotent radical of $P$.

Let $R \in \operatorname{Syl}_{r}(H)$; then $R^{\prime}=Z(R)$. Observe first that the elements of $R^{\prime}$ are conjugate with those in $Z(U)$. This follows from a result of Kantor [34, p. 377], stated that $H$ is generated by some subgroups of $G$ conjugate to $Z(U)=U_{3 \alpha+2 \beta}(q)$. Indeed, if $r \neq 2$ then in a 7-dimensional representation $\rho$, say, of $G$ over $\overline{\mathbb{F}}_{r}$ the elements of $Z(U)$ satisfy the equation $(x-\mathrm{Id})^{2}=0$; this is the case only for elements of $R^{\prime}$ in $R$ as $\rho(M)$ is a direct sum of two representations of degree 3 and the trivial one 34. If $r=2$ then the elements of $Z(R)$ are involutions, whereas all involutions of $R$ lie in $R^{\prime}$. It follows that a subgroup of $H$ conjugate with $Z(U)$ is conjugate in $H$ to $Z(R)$.

Therefore, we may assume that $R^{\prime}=Z(U)$. Let $B=N_{H}\left(R^{\prime}\right)$, so that $B$ is a Borel subgroup of $H$, and $|B|=q^{3}\left(q^{2}-1\right)$. One observes that $B$, acting on $R / R^{\prime}$ by conjugation, either permutes transitively the non-identity elements of $R / R^{\prime}$, or has 3 orbits of the same length $\left(q^{2}-1\right) / 3$ (in the latter case $\left.Z(H) \cong C_{3}\right)$. In particular, the only $B$-invariant subgroups of $R$ that contain $R^{\prime}$ are $R^{\prime}$ or $R$. Now, as $U=O_{r}(P)$ and $B=P \cap H$, we have that $U \cap B \leq O_{r}(B)=R$ and $U \cap B \triangleleft B$. If moreover $U \cap B=R^{\prime}$, then $|B U / U|=\left|B / R^{\prime}\right|$ has order $q^{2}\left(q^{2}-1\right)$, where Sylow $r$-subgroups of $P / U$ have order $q$, a contradiction. Hence $U \cap B=R$.

Suppose the contrary, that every irreducible constituent of $\left.V\right|_{H}$ is either trivial module or a Weil module. By [23, Lemma 11.1], for a Weil module $L$, say, the restriction $\left.L\right|_{R}$ contains no nontrivial linear character of $R$. So the same is true for $\left.V\right|_{R}$ and thus $R$ acts trivially on $C_{V}(Z(U))$. However, this contradicts Lemma 7.11 as $Z(U)<R<U$.

Even though $G=G_{2}(2)$ is not simple, we still consider it for completeness. Note that it contains a normal subgroup $H \cong \mathrm{SU}_{3}(3)$ of index 2 .

Lemma 7.13. Let $G=G_{2}(2)$ and let $g \in G$ be a p-element for an odd prime $p$. Let $\phi \in \operatorname{Irr}_{\ell} G$ for $\ell>2$. Suppose that $\operatorname{deg} \phi(g)<|g|$. Then $|g| \in\{3,7\}, \operatorname{dim} \phi=6$ and $\operatorname{deg} \phi(g)=|g|-1$.

Proof. Note that $|G|=2^{6} \cdot 3^{3} \cdot 7$ so $p=7$ or 3 . If $p \neq \ell$ then the result follows by inspection of the Brauer character tables. Let $p=\ell$.

If $p=7$ then Sylow 7-subgroups of $G$ are cyclic. By [60, Lemma 3.3(v)], if $\operatorname{deg} \phi(g)<|g|$ then $\operatorname{dim} \phi \leq 6$. As $g \in H \cong \mathrm{SU}_{3}(3)$, the result follows from the main theorem of [60].

Let $|g|=3$ and $\ell=3$. If $\operatorname{dim} \phi=6$ then $\left.\phi\right|_{H}$ is a direct sum of two irreducible representations of degree 3, which are Galois conjugate to each other. It follows that the Jordan form of $\phi(g)$ is $\operatorname{diag}\left(J_{2}, J_{2}, 1,1\right)$. Suppose that $\operatorname{dim} \phi>6$. By Clifford's theorem, $\left.\phi\right|_{H}$ is either irreducible or a direct sum of two irreducible representations of equal degrees. It follows that there exists a 3-modular irreducible representation $\tau$ of $H$ of degree $d>3$ such that $\operatorname{deg} \tau(g)=2$. This contradicts a result of [47, Theorem 1].

Lemma 7.14. Let $G=2 \cdot G_{2}(4), 1_{G} \neq \phi \in \operatorname{Irr}_{\ell} G$ for $\ell \neq 2$ and let $g \in G$ be a p-element of $G$ for $p>2$. Suppose that $\operatorname{deg} \phi(g)<|g|$. Then $\operatorname{dim} \phi=12,|g| \in\{3,7,13\}$ and $\operatorname{deg} \phi(g)=|g|-1$.

Proof. If $\ell \neq p$ then the result follows from the Brauer character table of $G$ [33. Let $\ell=p$. If $|g| \in\{7,13\}$ then Sylow $p$-subgroups of $G$ are cyclic and the result is contained in [60]. We are left with $p \in\{3,5\}$ and $|g|=p$. Let $|g|=3$. If $g \in 3 B$ then $g$ is contained in a subgroup $X \cong \mathrm{PSL}_{2}(13)$, so $\operatorname{deg} \phi(g)=3$ for every $1_{G} \neq \phi \in \operatorname{Irr}_{3} G$ as this holds for $X$. Suppose that 
$g \in 3 A$. As $g$ has just two distinct eigenvalues in an irreducible representation $\tau$ of $G$ of degree 12 over $\mathbb{C}$, the minimum polynomial degree of $g$ in the reduction of this modulo 3 equals 2 as well.

Suppose that $\operatorname{dim} \phi>12$. Note that $G / Z(G)$ contains a subgroup $Y \cong \mathrm{SU}_{3}(3)=G_{2}(2)^{\prime}$. As the Schur multiplier of $Y$ is trivial, we can assume that $Y$ is a subgroup of $G$. As $|Y|_{3}=|G|_{3}=$ 27 , we observe that $g$ is conjugate to an element of $Y$. We claim that the non-trivial composition factors of $\left.\phi\right|_{Y}$ are of dimension 3. Indeed, if $\lambda \in \operatorname{Irr}_{3}(Y)$ is such a factor then $\operatorname{deg} \lambda(g)=2$. Then $\lambda$ extends to a representation $\bar{\lambda}$ of $\mathrm{SL}_{3}\left(\overline{\mathbb{F}}_{3}\right)$. By [47], $\bar{\lambda}$ is a Frobenius twist of an irreducible representation with highest weight $\omega_{1}$ or $\omega_{2}$, which are the fundamental weights of the weight system of $\mathrm{SL}_{3}\left(\overline{\mathbb{F}}_{3}\right)$. These representations are of dimension 3, whence the claim. Note that $Y$ itself has two irreducible representation over $\overline{\mathbb{F}}_{3}$ dual to each other, and we denote their Brauer characters by $\lambda_{1}, \lambda_{2}$.

Let $h \in G$ be of order 7 . There is a single conjugacy class of such elements, so $h$ is rational and hence $\beta(h)$ is an integer, where $\beta$ is the Brauer character of $\phi$. It follows that $\left.\beta\right|_{Y}=$ $a\left(\lambda_{1}+\lambda_{2}\right)+b \cdot 1_{Y}$, so $\beta(1)=6 a+b$. Therefore, $\beta(h)=-a+b$, whence $a=(\beta(1)-\beta(h)) / 7$, $b=a+\beta(h)$.

In view of Theorem 7.1, $\phi$ is faithful. Furthermore, $Y$ has a unique involution $z$, say, and $\lambda_{1}(z)=\lambda_{2}(z)=-1$. Therefore, $\beta(z)=-2 a+b$. We use the Brauer character table of $G$ for $\ell=3$ in [33, p. 274]. Note that $z$ is in class $2 A$ in $G / Z(G)$, as $\beta(z)=-4$ if $\beta(1)=12$. By [33, p. 274], we have

\begin{tabular}{|c|c|c|c|c|c|c|c|c|c|}
\hline$\beta(1)$ & 12 & 104 & 352 & 1260 & 1364 & 1800 & 2016 & 3744 & 3888 \\
\hline$\beta(h)$ & -2 & -1 & 2 & 0 & -1 & 1 & 0 & -1 & 3 \\
\hline$\beta(z)$ & -4 & 8 & -32 & -36 & -28 & 40 & 96 & 32 & -16 \\
\hline$\beta(1)-\beta(h)$ & 14 & 105 & 350 & 1260 & 1365 & 1799 & 2016 & 3745 & 3885 \\
\hline$a$ & 2 & 15 & 50 & 180 & 195 & 257 & 288 & 535 & 555 \\
\hline$b$ & 0 & 14 & 52 & 180 & 194 & 258 & 288 & 534 & 558 \\
\hline
\end{tabular}

It is obvious that the relation $\beta(z)=-2 a+b$ holds only for $\beta(1)=12$.

Let $|g|=5$. Note that a subgroup $H \cong \mathrm{SU}_{3}(4)$ contains a Sylow 5-subgroup of $G$. The irreducible representation of $G$ of degree 12 (in any characteristic) remains irreducible under restriction to $H$, and this is a Weil representation of $H$. By [55, Lemma 6.1], $\operatorname{deg} \phi(g)=4$ if $\phi$ is a Weil representation of degree 12 .

Let $\phi \in \operatorname{Irr}_{5}(G)$ and $\phi(1)>12$. Then $\phi(1) \neq 1800,3600,3900$ as these characters are of 5defect 0 . In notation of [33], we are to inspect the characters $\phi_{i}$ with $i=22,23,24,25,28,29,30$.

By [55, Lemma 6.1], every non-trivial irreducible constituent of $\left.\phi\right|_{H}$ is of degree 12 . So $\left.\phi\right|_{H}=a \cdot \tau+b \cdot 1_{G}$, where $\tau \in \operatorname{Irr}_{5}(H)$ with $\tau(1)=12$. Then $\phi(1)=12 a+b$. Let $g_{13} \in H$ be of order 13. Then $\tau\left(g_{13}\right)=-1$, so $\phi\left(g_{13}\right)=b-a$. In particular, $\phi\left(g_{13}\right)$ is an integer, and the Brauer character table for $\ell=5$ shows that $\phi\left(g_{13}\right) \in\{0,1,-1\}$. Let $g_{2}$ be of order 2 then $\tau\left(g_{2}\right)=-4$, whence $\phi\left(g_{2}\right)=-4 a+b$.

If $a=b$ so $\phi\left(g_{13}\right)=0$ and $\phi(1)=13 a$, so $13 \mid \phi(1)$, whence $i=23,28,30$. In addition, $\phi\left(g_{2}\right)=-3 a$ so $3 \mid \phi(1)$, which is false for $i=23,28,30$.

Let $b=a+1$. Then $\phi\left(g_{13}\right)=1$, whence $i=22,24,29$. As $\phi(1)=13 a+1$, we have $a=7,43,167$, resp. In addition, $\phi\left(g_{2}\right)=-3 a+1=-20,-128,500$, which is false.

Let $b=a-1$. Then $\phi\left(g_{13}\right)=-1$, whence $i=25$. Then $\phi(1)=1260=13 a-1$, whence $a=97$. Then $\phi\left(g_{2}\right)=-3 a-1=-592$, which is false again.

\section{The CASE OF ${ }^{3} D_{4}(q)$}

In this section we consider the groups $G={ }^{3} D_{4}(q)$ and prove the following result.

Theorem 8.1. Theorem 1.1 is true for groups of type ${ }^{3} D_{4}(q)$.

We first consider the case where Sylow $p$-subgroups are cyclic and next the remaining cases. 
8.1. The case of cyclic Sylow $p$-subgroups. Note that $G$ contains a cyclic torus of order $q^{4}-q^{2}+1$. As $|T|$ is coprime to $|G| /|T|$, the Sylow $p$-subgroups of $T$ and of $G$ are cyclic for $p$ dividing $|T|$, and $C_{G}(t)=T$ for every $1 \neq t \in T$, that is, $t$ is regular. So the assumptions of Lemmas 3.2 and 3.7 hold. Therefore, if $\phi \in \operatorname{Irr}_{\ell} G$ then either $\left.\phi\right|_{T}=k \cdot \rho_{T}^{\text {reg }}$ with $k>0$ or $\phi$ is liftable or $\phi$ is unipotent. If $\ell \in\{0, p\}$ then Theorem 8.1 follows from [60], so we assume $\ell \neq 0, p$. In addition, we can assume that $\phi$ is not liftable. By Lemma 3.7, we are left with $\ell$-modular Brauer irreducible characters from the unipotent blocks, that is, we assume that $\phi$ is a constituent of a unipotent character modulo $\ell$.

We first specify Lemma 2.1 for our situation. Note that the degree of any non-trivial Brauer character of $G$ (provided $\ell$ is coprime to $q$ ) is at least $q^{5}-q^{3}$ [48, Table 1].

Lemma 8.2. In the notation of Lemma 2.1, let $C=T<G$ so that $|C|=q^{4}-q^{2}+1$. Let $1_{G} \neq \phi \in \operatorname{Irr}_{\ell} G$. Suppose that $\phi(g)=c<0$ for all $g \in C$, and that $-c<q$. Then $\left.\phi\right|_{C}-\rho_{C}^{\mathrm{reg}}$ is a proper character of $C$.

Proof. We have $-c(|C|-1)<q^{5}-q^{3} \leq \phi(1)$, so the result follows from Lemma 2.1 .

The $\ell$-decomposition matrix of $G$ is determined by Geck [18] for $\ell>2$ and Himstedt [26] for $\ell=2$, but a few entries for which only partial information has been obtained. For $\ell>3$ Dudas [12] has determined some of those entries. For undetermined entries we need upper bounds; for $\ell=2$ these are available from [26, and for $\ell>2$ these can be read off from the proof given in [18]. We acknowledge Dr. Himstedt's help with this matter.

There are 8 unipotent characters of $G$, denoted by $\mathbf{1},\left[\varepsilon_{1}\right],\left[\varepsilon_{2}\right],\left[\rho_{1}\right],\left[\rho_{2}\right]$, St, ${ }^{3} D_{4}[1],{ }^{3} D_{4}[-1]$ in [18] and elsewhere. We simplify this notation below by setting $D^{+}={ }^{3} D_{4}[1], D^{-}={ }^{3} D_{4}[-1]$ and using $1_{G}, \varepsilon_{1}, \varepsilon_{2}, \rho_{1}, \rho_{2}$ in place of $\mathbf{1},\left[\varepsilon_{1}\right],\left[\varepsilon_{2}\right],\left[\rho_{1}\right],\left[\rho_{2}\right]$.

We have $\varepsilon_{1}(1)=q\left(q^{4}-q^{2}+1\right), \varepsilon_{2}(1)=q^{7}\left(q^{4}-q^{2}+1\right)$, St $(1)=q^{12}, \rho_{1}(1)=q^{3}\left(q^{3}+1\right)^{2} / 2$, $\rho_{2}(1)=q^{3}(q+1)\left(q^{4}-q^{2}+1\right) / 2, D^{+}(1)=q^{3}(q-1)^{2}\left(q^{4}-q^{2}+1\right) / 2, D^{-}(1)=q^{3}\left(q^{3}-1\right)^{2} / 2$.

Furthermore $\varepsilon_{1}(1) \equiv 0(\bmod T), \varepsilon_{2}(1) \equiv 0(\bmod T), \rho_{2}(1) \equiv 0(\bmod T), D^{+}(1) \equiv 0$ $(\bmod T), \rho_{1}(1) \equiv-1(\bmod T), \operatorname{St}(1) \equiv 1(\bmod T), D^{-}(1) \equiv 1(\bmod T)$. This implies $\varepsilon_{1}(t)=$ $\varepsilon_{2}(t)=\rho_{2}(t)=D^{+}(t)=0, \rho_{1}(t)=-1, \operatorname{St}(t)=D^{-}(t)=1$ for $1 \neq t \in T$.

Himstedt [26] identifies the $\ell$-modular irreducible representations of $G$ of degree $<\left(q^{5}-q^{3}+\right.$ $q-1)^{2}$. As a consequence of this, we have

Lemma 8.3. Let $1_{G} \neq \phi \in \operatorname{Irr}_{\ell} G$. Then either $\phi(1)>q^{8}+q^{4}$ or $\phi$ is an irreducible constituent of $\varepsilon_{1}^{\circ}$ and $\phi(1)=\varepsilon_{1}(1)$ or $\varepsilon_{1}(1)-1$.

Let $T$ be a torus of order $q^{4}-q^{2}+1$ and $1 \neq t \in T$ is an arbitrary $\ell^{\prime}$-element. Using the data from [18] and [26], we will show that either $\phi(t) \geq 0$ or $-\phi(t) \cdot|T|<\phi(1)$ whenever $\phi \neq 1_{G}$ is a unipotent Brauer character of $G$. For this we first obtain an upper bound for $\phi(t)$.

Recall that $\operatorname{Irr}_{\ell}^{0}(G)$ denotes the set of non-liftable unipotent Brauer characters of $G$, and use $\phi_{i}$ with $1 \leq i \leq\left|\operatorname{Irr}_{\ell}^{0}(G)\right|$ to denote the Brauer characters in $\operatorname{Irr}_{\ell}^{0}(G)$. (This notation for $\phi_{i}$ does not coincide with the one used in [26].)

If $2,3 \neq \ell \mid(q-1)$ then $\left|\operatorname{Irr}_{\ell}^{0}(G)\right|=0$, so every irreducible $\ell$-modular character is liftable.

Let $\ell \mid\left(q^{4}-q^{2}+1\right)$. By [18, p. 3265], $\left|\operatorname{Irr}_{\ell}^{0}(G)\right|=2$, and $\phi_{1}=\rho_{1}-1_{G}, \phi_{2}=$ St $-\rho_{1}$. So $\phi_{1}(t)=-2$ and $\phi_{2}(t)=2$.

Let $2,3 \neq \ell \mid(q+1)$. Then $\left|\operatorname{Irr}_{\ell}^{0}(G)\right|=3$ and $\phi_{1}=\varepsilon_{1}-1_{G}, \phi_{2}=\varepsilon_{2}-1_{G}, \phi_{3}=\mathrm{St}-\varepsilon_{1}-\varepsilon_{2}-$ $a D^{-}-b D^{+}$, where $1 \leq a, b \leq(q-1) / 2$. (In fact, $a=b=2$ unless possibly $\ell=5$ and $q+1$ is not a multiple of 25 [12, Theorem 2.3]). Then $\phi_{1}(t)=\phi_{2}(t)=-1, \phi_{3}(t)=1-a$ so $-\phi_{3}(t)<q$.

Let $3 \neq \ell \mid\left(q^{2}+q+1\right)$. Then $\left|\operatorname{Irr}_{\ell}^{0}(G)\right|=4$ and $\phi_{1}=\rho_{1}-\varepsilon_{1}, \phi_{2}=\rho_{2}-1_{G}, \phi_{3}=\varepsilon_{2}-\rho_{1}+\varepsilon_{1}-a D^{+}$, $\phi_{4}=\mathrm{St}-c \phi_{3}-b D^{+}-\phi_{2}=\mathrm{St}-c \varepsilon_{2}+c \rho_{1}-c \varepsilon_{1}-a c D^{+}-\rho_{2}+1_{G}-b D^{+}=\mathrm{St}-c \varepsilon_{2}+c \rho_{1}-$ $c \varepsilon_{1}-(b+a c) D^{+}-\rho_{2}+1_{G}$.

So $\phi_{1}(t)=\phi_{2}(t)=-1, \phi_{3}(t)=1, \phi_{4}(t)=2-c$. In fact, $c=2$ by [12, Theorem 2.4], so $\phi_{4}(t)=0$. 
Let $3 \neq \ell \mid\left(q^{2}-q+1\right)$. Then $\left|\operatorname{Irr}_{\ell}^{0}(G)\right|=3$ and $\phi_{1}=\rho_{2}-\varepsilon_{1}-1_{G}, \phi_{2}=\varepsilon_{2}-a D^{-}-\phi_{1}-1_{G}=$ $\varepsilon_{2}-a D^{-}-\rho_{2}+\varepsilon_{1}, \phi_{3}=\mathrm{St}-d \phi_{2}-c D^{+}-b D^{-}-\phi_{1}-\varepsilon_{1}=\mathrm{St}-d\left(\varepsilon_{2}-a D^{-}+\varepsilon_{1}+1_{G}\right)-c D^{+}-b D^{-}-$ $\left(\varepsilon_{1}-1_{G}\right)-\varepsilon_{1}=\mathrm{St}-d \phi_{2}-c D^{+}-b D^{-} \phi_{1}-\varepsilon_{1}=\mathrm{St}-d \varepsilon_{2}+(a d-b) D^{-}+d \varepsilon_{1}+(1-d) \cdot 1_{G}-c D^{+}$.

So $\phi_{1}(t)=-1, \phi_{2}(t)=-a, \phi_{3}(t)=2+a d-b$. Here $a=d=0, b=2$ by [12, Theorem 2.5], so $\phi_{3}(t)=0$.

Let $\ell=3 \mid(q-1)$. Then $\left|\operatorname{Irr}_{\ell}^{0}(G)\right|=4$ and $\phi_{1}=\rho_{1}-\varepsilon_{1}, \phi_{2}=\rho_{2}-1_{G}, \phi_{3}=\varepsilon_{2}-\phi_{1}-a D^{+}=$ $\varepsilon_{2}-\rho_{2}+\varepsilon_{1}-a D^{+}, \phi_{4}=\mathrm{St}-c \phi_{3}-b D^{+}-\phi_{2}=\mathrm{St}-c\left(\varepsilon_{2}-\rho_{2}+\varepsilon_{1}-a D^{+}\right)-b D^{+}-\rho_{2}+1_{G}=$ $\mathrm{St}-c\left(\varepsilon_{1}+\varepsilon_{2}\right)+(c-1) \rho_{2}+(a c-b) D^{+}+1_{G}$.

So $\phi_{1}(t)=\phi_{2}(t)=-1, \phi_{3}(t)=1, \phi_{4}(t)=2-c$. Here $c \leq q$, so $-\phi_{4}(t)<q$.

Let $\ell=3 \mid(q+1)$. Then $\left|\operatorname{Irr}_{\ell}^{0}(G)\right|=4$ and $\phi_{1}=\varepsilon_{1}-1_{G}, \phi_{2}=\rho_{2}-\phi_{1}-2.1_{G}, \phi_{3}=$ $\varepsilon_{2}-\phi_{2}-a D^{-}-1_{G}, \phi_{4}=\mathrm{St}-d \phi_{3}-c D^{+}-b D^{-}-\phi_{2}-\phi_{1}+1_{G}=\mathrm{St}-d \phi_{3}-c D^{+}-b D^{-}-\rho_{2}(1)$, as $\phi_{1}+\phi_{2}=\rho_{2}-2.1_{G}$.

So $\phi_{1}(t)=\phi_{2}(t)=-1, \phi_{3}(t)=-a, \phi_{4}(t)=2+a d-b$.

In this case $0 \leq a \leq 1, a+1 \leq b \leq 3(q+1) / 2, c \leq(q-1) / 2$ and $1 \leq d \leq q$ [18. So $-\phi_{4}(t) \leq \frac{3 q-1}{2}$.

Let $\ell=2$. Then by [26, Theorem 3.1, p.572], we have $\left|\operatorname{Irr}_{\ell}^{0}(G)\right|=5$ and $\phi_{1}=\varepsilon_{1}-1_{G}$, $\phi_{2}=\rho_{1}-D^{+}, \phi_{3}=\rho_{2}-\phi_{2}, \phi_{4}=\varepsilon_{2}-1_{G}, \phi_{5}=\mathrm{St}-\phi_{4}-a D^{+}-b \phi_{3}-\phi_{1}-1_{G}$, where $0 \leq a, b \leq q$. We have $\phi_{1}(t)=\phi_{2}(t)=\phi_{4}(t)=-1, \phi_{3}(t)=1, \phi_{5}(t)=2-b$.

Lemma 8.4. Let $1_{G} \neq \phi \in \operatorname{Irr}_{\ell}^{0} G$ and $T$ be a torus of order $q^{4}-q^{2}+1$ of $G$. Then either $\phi(t)>0$ or $-\phi(t) \cdot|T|<\phi(1)$ for every $1 \neq t \in T$. In particular, $\operatorname{deg} \phi(t)=|t|$ for any $p$-element $t \in T$ with $p \neq \ell$.

Proof. Suppose first that $\phi$ is a non-trivial irreducible constituent of $\varepsilon_{1}$. Then $\phi(1)=\varepsilon_{1}(1)-1$ (and either $\ell=2$ or $\ell=3 \mid(q+1)$ ). Then $\phi(t)=-1$ and $\phi(1)=q\left(q^{4}-q^{2}+1\right)-1$, whence the claim.

Suppose that $\phi$ is not a constituent of $\varepsilon_{1}$. Then $\phi(1)>q^{8}+q^{4}$ by Lemma 8.3 , and either $\phi(t)>0$ or $-\phi(t) \leq(3 q-1) / 2$. Then $|T| \cdot(3 q-1) / 2=\left(q^{4}-q^{2}+1\right)(3 q-1) / 2<q^{8}+q^{4}$.

For the last claim, observe by Lemma 3.7 that $\phi$ takes a constant value $c$ on $\langle t\rangle \backslash\{1\}$. Now apply Lemma 2.1.

Remark 8.5. In [26] there are weaker bounds for $a, b, c, d$ for $3=\ell \mid(q+1)$, specifically $a \leq$ $q(q-1), b \leq\left(q^{3}-1\right) / 2, c, d \leq(q-1) / 2$. These are sufficient for our purpose, as either $\phi(t)>1$ or $-\phi_{4}(t) \leq\left(\left(q^{3}-1\right) / 2\right)-2$, and again we have $q^{3}|T|<\phi_{4}(1)$.

8.2. The case where Sylow $p$-subgroups are not cyclic. For uniformity we denote by $\mathrm{SO}_{2 n}\left(\overline{\mathbb{F}}_{q}\right)$ the subgroup of index 2 of $O_{2 n}\left(\overline{\mathbb{F}}_{q}\right)$ if $q$ is odd and of $O_{2 n}\left(\overline{\mathbb{F}}_{q}\right)$ if $q$ is even. Then $\mathrm{SO}_{2 n}\left(\overline{\mathbb{F}}_{q}\right)$ is a connected simple algebraic group of type $D_{n}$ If $q$ is even then $S O_{2 n}\left(\overline{\mathbb{F}}_{q}\right)$ is formed by elements of quasi-determinant 1 in $O_{2 n}\left(\overline{\mathbb{F}}_{q}\right)$ Also, let $\mathbf{G}=\operatorname{Spin}_{2 n}\left(\overline{\mathbb{F}}_{q}\right)$ denote the simply connected simple algebraic group of type $D_{n}$, so that $\mathbf{G}=\mathrm{SO}_{2 n}\left(\overline{\mathbb{F}}_{q}\right)$ when $2 \mid q$ and $\mathbf{G} / C_{2}=\mathrm{SO}_{2 n}\left(\overline{\mathbb{F}}_{q}\right)$ when $2 \nmid q$. Taking $n=4$, we can view $G=\mathbf{G}^{F}$ for some Steinberg endomorphism $F: \mathbf{G} \rightarrow \mathbf{G}$. By [36, p. 33], $G \cong{ }^{3} D_{4}(q)$ has maximal subgroups isomorphic to $G_{2}(q)$ and $\left(C_{q^{2}+q+1} \circ \mathrm{SL}_{3}(q)\right) \cdot \operatorname{gcd}(3, q-1)$ (where $C_{q^{2}+q+1}$ is cyclic of order $q^{2}+q+1$ ). If $g \in G$ lies in the $G_{2}(q)$-subgroup then we can use our result on $G_{2}(q)$ (Theorem 7.1). So our first goal is to establish Lemma 8.9 below.

Lemma 8.6. Let $\mathbf{G}$ be a simple, simply connected algebraic group of type $D_{4}$ in defining characteristic $r$, and let $V$ be the standard $\mathbf{G}$-module. Let $G \cong{ }^{3} D_{4}(q)<\mathbf{G}$, where $r \mid q$ and $q>2$. Let $M_{1} \cong\left(C_{q^{2}+\varepsilon q+1} \circ \mathrm{SL}_{3}^{\varepsilon}(q)\right) \cdot \operatorname{gcd}(3, q-\varepsilon)$ and $M_{2} \cong G_{2}(q)$ be maximal subgroups of $G$ and $H_{1}<M_{1}$ a subgroup isomorphic to $\mathrm{SL}_{3}^{\varepsilon}(q)$. Then $H_{1}$ is $\mathbf{G}$-conjugate to a subgroup of $M_{2}$.

Proof. Note that $V$ is self-dual. Our strategy is to show that for some subgroup $H_{2} \cong \mathrm{SL}_{3}^{\varepsilon}(q)$ of $M_{2}=G_{2}(q)$ for every $i=1,2$ there are $H_{i}$-stable subspaces $V_{1}^{i}, V_{2}^{i}, V_{3}^{i}$ such that $V=V_{1}^{i} \oplus V_{2}^{i} \oplus V_{3}^{i}$, $V_{1}^{i}, V_{2}^{i}$ are totally singular of dimension 3 , and $V_{3}^{i}$ is non-degenerate and trivial on $H_{i}$. Then 
$V_{1}^{i}, V_{2}^{i}$ are dual $F H_{i}$-modules, and the result will follow from Witt's theorem. Indeed, by Witt's theorem applied to $\mathrm{O}(V)$, there is some $x \in \mathrm{O}(V)$ that sends $V_{j}^{1}$ to $V_{j}^{2}$ for $j=1,2,3$. In our case $V_{3}^{i}$ is a trivial $H_{i}$-module of dimension 2 and so we can find an element $y \in\left(\mathrm{O}\left(V_{3}^{2}\right) \backslash \mathrm{SO}\left(V_{3}^{2}\right)\right)$ that commutes with $H_{2}$. Replacing $x$ by $y x$ if necessary, we may assume $x \in \mathrm{SO}(V)$ and sends $V_{j}^{1}$ to $V_{j}^{2}$. Since all 3-dimensional nontrivial representations of $H_{i}$ are irreducible and quasiequivalent to the natural representation, we now have that $x$ sends the image of $H_{1}$ in $\mathrm{SO}(V)$ to the image of $H_{2}$ in $\mathrm{SO}(V)$, and we are done if $r=2$. If $r>2$, then an inverse image of $x$ conjugates the full inverse image $C_{2} \times H_{1}$ to $C_{2} \times H_{2}$, hence $H_{1}$ to $H_{2}$.

If $r \neq 3$ then the subgroups of $M_{2}$ isomorphic to $\mathrm{SL}_{3}^{\varepsilon}(q)$ are conjugate so choose for $H_{2}$ any of them. If $r=3$ then $G_{2}$ has two conjugacy classes of subgroups isomorphic to $\mathrm{SL}_{3}^{\varepsilon}(q)$, one of which is reducible on the irreducible $F M_{2}$-modules of dimension 7 , and the other is irreducible [36. Theorem A]. In this case we choose $H_{2}$ from the former one.

It suffices to show that the composition factors of $\left.V\right|_{H_{i}}, i=1,2$ are of dimension 1 or 3 . Indeed, as $V$ is self-dual and the simple $H_{i}$-modules of dimension 3 are not self-dual, there are two composition factors of dimension 3 and they are dual to each other. Let $N$ be a composition factor of dimension 3. It suffices to observe that $\operatorname{Ext}_{H_{i}}^{1}\left(N, N^{*}\right)=0$ and $\operatorname{Ext}_{H_{i}}^{1}\left(N, N_{0}\right)=0=$ $\operatorname{Ext}_{H_{i}}^{1}\left(N_{0}, N\right)$, where $N_{0}$ is the trivial $F H_{i}$-module and $N^{\prime}$ is dual to $N$. If $r>2$ then every $\mathrm{FH}$-module of dimension almost 6 is completely reducible by [43, Theorem 1.1]. If $r=2$ then this follows by [51].

Suppose first that $i=2$. As the dimensions at most 8 of nontrivial simple $G_{2}(q)$-modules are 6 if $r=2$ and 7 if $r \neq 2$ [40, p. 167], the fixed point subspace $L$ of $M_{2}$ on $V$ is non-zero. Next, if $r \neq 2$ then $K$ must be non-degenerate, and $V=L \oplus V^{\prime}$, where $V^{\prime}$ is an irreducible $F M_{2}$-module. If $r=2$ then as $\operatorname{dim} V / L=1, V / L$ is reducible, and has a composition factor $V_{1}^{\prime}$ of degree 6 . Note that the irreducible constituents of the restrictions of these modules $V^{\prime}$, respectively $V_{1}^{\prime}$ to $\mathrm{H}_{2}$ are 3-dimensional and dual to each other. (Indeed, if $r=2$ then all nontrivial simple $H_{2}$-modules of dimension $\leq 6$ are of dimension 3 and non-self-dual, see [40, p. 149]. If $r \neq 2$, then by the choice of $H_{2}, H_{2}$ is reducible on $V^{\prime}$. If moreover it has a composition factor $W$ of dimension $\neq 1,3$ on $V^{\prime}$, then $\operatorname{dim} W=6$ by [40, p. 149] and $W$ is not self-dual. The other composition factors of the $H_{2}$-modules are all trivial, and this contradicts $V \cong V^{*}$.)

Suppose that $i=1$. Let $K$ be an irreducible $F H_{1}$-submodule of $V$ of maximal dimension. Then $K \neq V$ by Schur's lemma. Suppose that $\operatorname{dim} K=7$. By [40, p. 149], we have $r=3$ and $V$ has a composition factor $K^{\prime}$ of dimension 1 . As $V$ is self-dual, we conclude that $V$ is completely reducible over $H_{1}$, so $K$ is non-degenerate, $V=K \oplus K^{\perp}$ and $\operatorname{dim} K^{\perp}=1$. Now the odd-order subgroup $C_{q^{2}+\varepsilon q+1}$ must act trivially on both $K$ and $K^{\perp}$, a contradiction. Suppose that $\operatorname{dim} K=6$. Then $r>2$ and $K$ is not self-dual [40, p.149], so $V_{H_{1}}$ has a composition factor dual to $K$, which is not the case by dimension reason. So $\operatorname{dim} K \leq 5$ and hence $\operatorname{dim} K=3$ by [40, p. 149].

Lemma 8.7. Let $H \cong \mathrm{SL}_{3}^{\varepsilon}(q) \subset \mathbf{G}=\operatorname{Spin}_{8}\left(\overline{\mathbb{F}}_{q}\right)$ and $V$ the natural module for $\mathrm{SO}_{8}\left(\overline{\mathbb{F}}_{q}\right)$. Suppose that $\left.V\right|_{H}=V_{1} \oplus V_{2} \oplus V_{3}$ with $V_{1}, V_{2}$ totally singular of dimension 3 and $V_{3}$ a non-degenerate subspace on which $H$ acts trivially. Then $Y:=C_{\mathbf{G}}(H)$ is connected.

Proof. By assumption, $V_{1}, V_{2}$ are dual to each other and $V_{3}$ is trivial as $H$-modules. Hence $Y$ stabilizes these modules; let $\bar{Y}$ denote the image of $Y$ in $\operatorname{SO}(V)$. Then $\bar{Y} \leq\left\{\operatorname{diag}\left(y_{1}, y_{2}, t\right)\right\}$, where $y_{1} \in \mathrm{GL}_{3}\left(\overline{\mathbb{F}}_{q}\right)$ is a scalar $(3 \times 3)$-matrix, $y_{2}=y_{1}^{-1}$ and $t \in \mathrm{O}\left(V_{3}\right)$. In fact, $t \in \mathrm{SO}\left(V_{3}\right)$. This is obvious if $q$ is odd; if $q$ is even then, since $\mathrm{GL}_{3}\left(\overline{\mathbb{F}}_{q}\right)$ has no subgroup of index 2 , so the matrix $\operatorname{diag}\left(y_{1}, y_{1}^{-1}, \operatorname{Id}_{2}\right)$ lies in $\mathrm{SO}(V)$, whence $\operatorname{diag}\left(\operatorname{Id}_{6}, t\right) \in \mathrm{SO}(V)$, which implies the claim. Note that $C_{\mathrm{SO}(V)}(H)$ contains the subgroup $\operatorname{diag}\left(\operatorname{Id}_{3}, \operatorname{Id}_{3}, \mathrm{SO}\left(V_{3}\right)\right)$. Hence $C_{\mathrm{SO}(V)}(H)$ is isomorphic to the direct product of $Z\left(\mathrm{GL}_{3}\left(\overline{\mathbb{F}}_{q}\right)\right) \cong \overline{\mathbb{F}}_{q}^{\times}$and $\mathrm{SO}_{2}\left(\overline{\mathbb{F}}_{q}\right)$, which is a connected group. Now, if $r=2$ then $Y=\bar{Y}=C_{\mathrm{SO}(V)}(H)$, and we are done. If $r>2$, then these two subgroups lift to a one-dimensional torus $T$ and $S=\mathrm{Spin}_{2}$, respectively, which centralize each other modulo $C_{2}=Z(\operatorname{Spin}(V))$. Since $S$ is perfect, we have that $[T, S]=[T,[S, S]]$ is contained 
in $[[T, S], S]=1$, so the full inverse image of $C_{\mathrm{SO}(V)}(H)$ is a central product of two connected subgroups, and so is connected. Each of $T$ and $S$ centralizes the perfect subgroup $H$ modulo $Z(\operatorname{Spin}(V))$, so the same argument shows that $Y$ is the full inverse image of $C_{\mathrm{SO}(V)}(H)$, and so $Y$ is connected.

Lemma 8.8. Let $\mathbf{G}$ be a connected algebraic group, $\mathbf{F}$ a Frobenius map, and let $G=\mathbf{G}^{\mathrm{F}}=\{g \in$ $\mathbf{G} \mid \mathbf{F}(g)=g\}$. Let $H$ be a subgroup of $G$ and $H_{1}=x H x^{-1} \leq G$ for some $x \in \mathbf{G}$. Suppose that $C_{\mathbf{G}}(H)$ is connected. Then $H, H_{1}$ are conjugate in $G$.

Proof. Let $h \in H$. Then $x h x^{-1} \in G$, so $x h x^{-1}=\mathrm{F}(x) h \mathrm{~F}(x)^{-1}$. Then $\mathrm{F}(x)^{-1} x \in C_{\mathbf{G}}(h)$ for all $h \in H$. Therefore, $\mathrm{F}(x)^{-1} x \in C_{\mathbf{G}}(H)$. By Lang's theorem, there exists $c \in C_{\mathbf{G}}(H)$ such that $\mathrm{F}(x)^{-1} x=\mathrm{F}(c)^{-1} c$, so $x c^{-1} \in G$ and $H_{1}=\left(x c^{-1}\right) H\left(x c^{-1}\right)^{-1}$.

Lemma 8.9. Let $H_{1}$ be as in Lemma 8.6. Then $H_{1}$ is $G$-conjugate to a subgroup of $M_{2} \cong G_{2}(q)$.

Proof. By Lemma 8.6, $x H_{1} x^{-1}<M_{2}<G$ for some $x \in \mathbf{G}$, so Lemma 8.8 yields the result.

Remark 8.10. Lemma 8.9 justifies the claim in [62, p. 2520, line 6] stated therein with no proof. Thus, this fixes a gap in the proof of [62, Lemma 4.14] which gives a proof of Theorem 8.1 for $\ell=0$ and $p>2$.

Proposition 8.11. Let $g \in G \cong{ }^{3} D_{4}(q)$ be a semisimple p-element. Suppose that Sylow $p$ subgroups of $G$ are not cyclic. Let $\theta \in \operatorname{Irr}_{F} G$ with $\operatorname{dim} \theta>1$. Then $\operatorname{deg} \theta(g)=|g|$.

Proof. (i) The case with $q=2$ can be settled by a computer computation. (Note that when $p \neq \ell$ one can also use the Brauer character table, and the $p=\ell>3$ case follows from [60]; so if $q=2$ then it suffices to deal with the case $p=\ell=3$.) Let $q>2$.

If $g$ is contained in a subgroup isomorphic to $G_{2}(q)$ then the lemma follows from Theorem 7.1. Suppose the opposite. Then $p$ divides the $r^{\prime}$-part of $|G| /\left|G_{2}(q)\right|=q^{6}\left(q^{8}+q^{4}+1\right)$, i.e. $p \mid\left(q^{8}+q^{4}+1\right)$. As we assume the Sylow $p$-subgroups of $G$ are non-cyclic, we have $p \mid\left(q^{4}+q^{2}+1\right)$, so $p$ divides $q^{2}+\varepsilon q+1$ for some $\varepsilon \in\{ \pm 1\}$. In particular, $p>2$.

(ii) Here we consider the case $p>3$. By [36], $G$ contains a subgroup $H$ isomorphic to $X \circ Y$, where $X \cong \mathrm{SL}_{3}^{\varepsilon}(q)$ and $Y \cong C_{\varepsilon}$, a cyclic subgroup of order $q^{2}+\varepsilon q+1$. Then

$$
|G| /|H|=\operatorname{gcd}(3, q-\varepsilon) q^{9}\left(q^{3}+\varepsilon\right)\left(q^{8}+q^{4}+1\right) /\left(q^{2}+\varepsilon q+1\right),
$$

whence $(p,|G| /|H|)=1$. Therefore, $H$ contains a Sylow $p$-subgroup of $G$. By Lemma 8.9, $X$ is contained in a subgroup $D \cong G_{2}(q)$.

Express $g=x y$ for $x \in X$ and $y \in Y$. Then $y \neq 1$ as otherwise $g \in X<D \cong G_{2}(q)$. In addition, by [?, Lemma 4.13], we may assume that $|x|=|y|=|g|$.

Suppose that $\operatorname{deg} \theta(g)<|g|$. Let $\tau$ be any composition factor of $\left.\theta\right|_{H}$ such that $\tau=\phi \otimes \lambda$ with $1_{X} \neq \phi \in \operatorname{Irr}_{F} X$ and $\lambda \in \operatorname{Irr}_{F} Y$. Then $\operatorname{deg} \phi(x)<|g|=|x|$. This implies that $\phi$ is a Weil representation of $X$ and $|g|=\left|C_{\varepsilon}\right|$, see Lemma 2.6 if $\varepsilon=1$ and [55, Proposition 6.1] if $\varepsilon=-1$. Therefore, every non-trivial composition factor of $\left.\theta\right|_{X}$ is a Weil representation of $X$. If $\varepsilon=-1$ and $(3, q)=1$ then this contradicts Lemma 7.12. If $3 \nmid(q-\varepsilon)$ and $\ell \mid\left(q^{4}+q^{2}+1\right)$ then this contradicts Lemma 7.9. Thus, if $\varepsilon=-1$, then we may assume $3 \mid q$ and $\ell \nmid\left(q^{4}+q^{2}+1\right)$, whence $p \neq \ell$. If $\varepsilon=1$, then, as $p>3$ and $q^{2}+q+1$ is a $p$-power, we have $3 \nmid(q-1)$, and so we may assume that $\ell \nmid\left(q^{4}+q^{2}+1\right)$, whence $p \neq \ell$. In both the cases, we have $Z(X)=1$ and $X \circ Y=X \times Y$.

We can write $\left.\tau\right|_{(X \times Y)}=\left(\chi_{1} \otimes \lambda_{1}\right)+\ldots+\left(\chi_{s} \otimes \lambda_{s}\right)$ as a sum of Brauer characters, where $\chi_{i} \in \operatorname{Irr}_{F} X$ and $\lambda_{i} \in \operatorname{Irr}_{F} Y$ for $i=1, \ldots, s$. We order the summands so that $\chi_{i}(1)>1$ for $i=1, \ldots, t \leq s$ and $\chi_{i}(1)=1$ for $i>t$. Let $\phi_{i}$ be the representation afforded by $\chi_{i}$, and let $\lambda_{i}$ also denote the respective representation of $Y$ as this character is linear. Then $\operatorname{deg} \phi_{i}(x)<|g|=|x|$. As $Z(X)=1$ and $\ell \neq p \mid\left(q^{2}+q \varepsilon+1\right)$, every non-trivial $p$-element is regular in $X$, so, by Lemma 3.7. either $\phi$ is liftable or constant on the non-identity $p$-elements of $X$. By Lemma 2.6 and [55, Proposition 6.1], if $1 \leq i \leq t$ then $\operatorname{deg} \phi_{i}(x)=|x|-1=\operatorname{dim} \phi_{i}(1), \phi_{i}$ is real, and hence $\operatorname{deg} \theta(g)=|g|-1$. Then $1 \notin \operatorname{Spec} \theta(g)$. (Indeed, otherwise some other $|g|$-root $\nu$ of unity is 
not in the spectrum of $\theta(g)$; as $p>2$ and $g$ is conjugate to $g^{-1}$ (see for instance [54, Theorem 1 (vi)]), $\nu^{-1} \notin \operatorname{Spec} \theta(g)$, and hence $\operatorname{deg} \theta(g) \leq|g|-2$, which is a contradiction). Since $\phi_{i}$ is real, we also have that $1 \notin \operatorname{Spec} \phi_{i}(x)$, and hence $\operatorname{Spec} \phi_{i}(x)$ consists of all nontrivial $|g|$-roots of unity when $1 \leq i \leq t$. It follows that $\lambda_{i}(y)=1$ for $1 \leq i \leq t$. As $y \notin \operatorname{ker}(\tau)$ and $1 \notin \operatorname{Spec} \theta(g)$, we have $s>t$, and none of $\lambda_{t+1}(y), \ldots, \lambda_{s}(y)$ equals 1 . It follows that 1 is not an eigenvalue of $\theta\left(x^{k} y^{l}\right)$ whenever $k, l$ are coprime to $p$, whereas $1 \in \operatorname{Spec} \theta(x)$. However, by [62, Lemma 4.13], $x$ is conjugate to some $x^{k} y^{l}$ with $\left|x^{k}\right|=\left|y^{l}\right|=|x|$ (the key point used in [62, Lemma 4.13] is that $N_{G}\left(T_{\varepsilon}\right)$ acts primitively on a maximal torus $\left.T_{\varepsilon} \cong C_{q^{2}+\varepsilon q+1}^{2}\right)$. This is a contradiction.

(iii) Let $p=3$. Then $|g|$ does not divide $q^{2}-1$. (Indeed, otherwise $|g|$ divides $q-\varepsilon$ for some $\varepsilon \in\{1,-1\}$, and, by Lemma 2.9 (i), $g$ is contained in a torus of $G$ of order $(q-\varepsilon)^{2}$. By Lemma 2.8 (iii), the tori of order $(q-\varepsilon)^{2}$ are conjugate in $G$. A subgroup isomorphic to $G_{2}$ contains a torus of this order, so it contains a conjugate of $g$.)

It is known (see claim $\left(^{*}\right)$ in the proof of [62, Proposition 4.8, p. 2517]) that $g$ is contained in a subgroup $H \cong X \circ Y$, where $X \cong \mathrm{SL}_{2}(q)$ and $Y \cong \mathrm{SL}_{2}\left(q^{3}\right)$. Express $g=x y$ with $x \in X$, $y \in Y$ to be 3-elements. Let $\tau$ be an irreducible constituent of $\left.\theta\right|_{H}$. Then $\tau=\phi \otimes \eta$, where $\phi, \eta$ are irreducible representations of $X, Y$, respectively. Hence $\tau(g)=\phi(x) \otimes \eta(y)$. Suppose that $\operatorname{deg} \theta(g)<|g|$. Then $\operatorname{deg} \tau(g)<|g|$ implies $\operatorname{deg} \phi(x)<|g|$ and $\operatorname{deg} \eta(y)<|g|$. If $|y| \leq|x|$ then $|g|$ divides $q^{2}-1$; by the above this is not the case. So $|y|>|x|$, and hence $|g|=|y|$. In this case, choose $\tau$ so that $\operatorname{dim} \eta>1$. Then $\operatorname{deg} \eta(y)<|g|=|y|$ implies by [11, Theorem 1.1] that $3 \mid(q+1)$. By [55, Lemma 3.3] applied to $Y$, it follows that $q^{3}+1$ is a 3-power if $q$ is even and $\left(q^{3}+1\right) / 2$ is a 3 -power if $q$ is odd. The former case is ruled out by Lemma 2.2 (as $\left.q>2\right)$. In the latter case $\left(q^{3}+1\right) / 2=\left(q^{2}-q+1\right)(q+1) / 2$ is a 3 -power implies $q^{2}-q+1$ and $(q+1) / 2$ to be 3 -powers, which is false as $\operatorname{gcd}\left(q^{2}-q+1,(q+1) / 2\right) \in\{1,3\}$.

Proof of Theorem 8.1. The result follows from Lemma 8.4 (and the discussion at the beginning of the section) if $p \mid\left(q^{4}-q^{2}+1\right)$, and from Proposition 8.11 if $p \nmid\left(q^{4}-q^{2}+1\right)$.

\section{The CASE $\mathrm{OF}^{2} F_{4}(q)$}

In this section we prove Theorem 1.1 for $G={ }^{2} F_{4}(q), q=2^{2 k+1}$.

Lemma 9.1. Theorem 1.1 is true for $G={ }^{2} F_{4}(2)^{\prime}$ or ${ }^{2} F_{4}(2)$.

Proof. If and $\ell \neq p$ then the result follows by inspection of the Brauer character table of $G$ [33. If $p=\ell=13$ then this follows from [60]. So we are left with $p=3,5$.

Note that $G$ has no element of order 9 or 25 and all elements of order 3 and of order 5 are conjugate. In addition, $G$ contains a subgroup $H \cong \mathrm{PSL}_{2}(25)$, so we can assume that $g \in H$. If $|g|=3$ then the result follows from [55, Lemma 3.3].

Let $|g|=5$. Then we can assume that $g$ is contained in a subgroup $K \cong \operatorname{PSL}_{2}(9)$. If $1_{K} \neq \tau \in \operatorname{Irr}_{5}(K)$ and $\operatorname{deg} \tau(g)<5$ then $\operatorname{dim} \tau=4$ ([55, Lemma 3.3]). Therefore, if the lemma is false then $\left.\phi\right|_{K}=a \tau+b \cdot 1_{G}$. Let $x \in K$ be of order 3 . Then $\tau(x)=2$, so $\phi(x)=2 a+b$, where $a \geq 1$. This implies $\phi(1) \leq 2 \phi(x)$, which contradicts the data in the Brauer character table of $G$ for $\ell=5$.

So in what follows we assume $q>2$. Observe that if Sylow $p$-subgroups of $G={ }^{2} F_{4}(q)$ are cyclic, then $p$ divides $q^{2}-q+1$ or $q^{4}-q^{2}+1$.

Lemma 9.2. Theorem 1.1 is true if is true if Sylow p-subgroups of $G$ are not cyclic.

Proof. Suppose the contrary, that is, $\operatorname{deg} \theta(g)<|g|$. As a Sylow $p$-subgroup is not cyclic, one observes that $p \mid\left(q^{4}-1\right)$. If $p \mid(q-1)$ or $p \mid\left(q^{2}+1\right)$ then $g$ is contained in a direct product of two copies of ${ }^{2} B_{2}(q)$ (see [62, Table 1]). In this case the result follows from that for ${ }^{2} B_{2}(q)$.

Suppose that $p \mid(q+1)$. If $p=3$ then $g$ is contained in a subgroup $H \cong \mathrm{SU}(3, q)$ [62, Table 1]. Then [55, Lemma 6.1] implies $q=8,|g|=9$ and $g$ is contained in a maximal torus of $H$ of order $(q+1)^{2}$. This is also true if $p>3$. This torus is also a maximal torus of a subgroup $H_{1} \cong \operatorname{Sp}_{4}(q)$. So we can assume $g \in H_{1}$, and the result follows from Lemma 2.5 . 
Lemma 9.3. Theorem 1.1 is true if $p \mid\left(q^{2}-q+1\right)$.

Proof. Suppose the contrary. As $q^{2}-q+1$ divides the order of a subgroup $H \cong \mathrm{SU}_{3}(q)$, it follows that $H$ contains a Sylow $p$-subgroup of $G$. Therefore, we assume that $g \in H$. By [55, Prop. 6.1(ii)], $3 \mid(q+1)$, which is impossible since $q=2^{2 m+1}$.

The above analysis reduces the proof of Theorem 1.1 to the case where $p \mid\left(q^{4}-q^{2}+1\right)$; in particular, the Sylow $p$-subgroups are cyclic. In view of [60] and [62], we may assume that $\ell \neq p$ and that $\phi$ is not liftable. Note that

$$
q^{4}-q^{2}+1=\left(q^{2}+q \sqrt{2 q}+q+\sqrt{2 q}+1\right)\left(q^{2}-q \sqrt{2 q}+q-\sqrt{2 q}+1\right),
$$

in fact, $G$ contains maximal tori $T_{1}, T_{2}$ of these orders so we may assume that $g \in T_{1}$ or $g \in T_{2}$. The rest of the section is therefore devoted to the proof of

Proposition 9.4. Theorem 1.1 is true if $p \mid\left(q^{4}-q^{2}+1\right)$ unless possibly when $\ell=3, q=8$, $p=109$ and $\phi=\phi_{21}$ in notation of [26].

First we recall

Lemma 9.5. Let $d_{\ell}$ be the minimum degree of a nontrivial $\ell$-modular irreducible representation of $G={ }^{2} F_{4}(q)$ with $q>2$.

(i) [26, Theorem 6.1] If $\ell>3$ then $d_{\ell} \geq(q-1)(q+1)^{2}\left(q^{2}-q+1\right) \sqrt{q / 2}$.

(ii) [56, Theorem 1.4] If $\ell=3$ then $d_{3} \geq(q-1)\left(q^{4}+q^{3}+q\right) \sqrt{q / 2}$.

Lemma 2.1 implies the following:

Lemma 9.6. Let $\phi$ be a non-trivial unipotent Brauer character of $G$, and $T \in\left\{T_{1}, T_{2}\right\}$. Let $T^{\prime}$ be the subgroup of $\ell^{\prime}$-elements of $T$ and $1 \neq t \in T^{\prime}$. If $\phi(t) \geq 0$ or $a=\phi(t)<0$ and $-a(|T|-1)<\phi(1)$ then $\left.\phi\right|_{T}$ contains every $\nu \in \operatorname{Irr} T$. In particular, this holds if $q>3, a<0$ and $-a<q\left(q^{3}+q^{2}+1\right) / 8$.

Proof. We have $|T|-1 \leq q^{2}+q+(q+1) \sqrt{2 q}$, so

and

$$
-a<\frac{(q-1)\left(q^{4}+q^{3}+q\right) \sqrt{q / 2}}{(q+1)(q+\sqrt{2 q})}=\frac{(q-1)\left(q^{4}+q^{3}+q\right)}{(q+1) 2+\sqrt{2 q})}
$$

as $q \geq 8$.

$$
\frac{q-1}{(q+1)(2+\sqrt{2 q})}>\frac{7}{9(2+\sqrt{16})}=\frac{7}{54}>1 / 8
$$

Recall that we have to deal only with the cases where $\ell \neq p$ and $\phi$ is not liftable. Therefore, Lemma 3.7 together with Lemma 9.6 reduces the proof to the case where $\phi$ is unipotent. Our strategy in proving Proposition 9.4 is to show, using the $\ell$-decomposition numbers of $G$, that either $\phi(t) \geq 0$ or $\phi(t)<q\left(q^{3}+q^{2}+1\right) / 8$, and then use Lemma 9.6.

Hiss [28] has determined the decomposition numbers of $G={ }^{2} F_{4}(q)$ modulo $\ell \mid\left(q^{4}-q^{2}+1\right)$ and $\ell \mid\left(q^{2}-q+1\right)$; Himstedt $[25]$ has computed these for remaining $\ell \neq 2$. Note that Himstedt's tables involve some indetermined values, which leads to certain difficulties below, in particular, for $q=8$.

The degrees of the unipotent characters are available in Malle [41. In his notation these are $\chi_{k}$ with $k=1, \ldots, 21$. Note that $G={ }^{2} F_{4}(q)$ has exactly two maximal tori $T_{1}, T_{2}$ (up to conjugation) that satisfies the assumption of Lemma 3.2. These are of the aforementioned orders $\left|T_{1}\right|=q^{2}+q \sqrt{2 q}+q+\sqrt{2 q}+1$ and $\left|T_{2}\right|=q^{2}-q \sqrt{2 q}+q-\sqrt{2 q}+1$. Let $\eta$ be as in Lemma 3.2. Then $\eta$ can be computed by taking the congruences of $\chi_{k}(1)$ modulo $\left|T_{i}\right|$ for $i=1,2$. The result is recorded in Hiss [25, p. 886 and p. 884], and we display it in Table 3 below. Note that fourth column lists the characters of $p_{i}$-defect 0 for $i=1,2$. 
Table 3: Unipotent character values at $1 \neq t \in\left(T_{1} \cup T_{2}\right)$

\begin{tabular}{|c|c|c|c|}
\hline & $\chi_{i}(t)=1$ & $\chi_{i}(t)=-1$ & $\chi_{i}(t)=0$ \\
\hline $1 \neq t \in T_{1}$ & $i=1,4,5,6,13,15,16,19,20$ & $i=7,8,10$ & $i=2,3,9,11,12,14,17,18,21$ \\
\hline $1 \neq t \in T_{2}$ & $i=1,4,7,8,12,17,18,19,20$ & $i=5,6,9$ & $i=2,3,10,11,13,14,15,16,21$ \\
\hline
\end{tabular}

In what follows, we will use data of Table 3 without further referring. Also, denote

$$
\begin{gathered}
\phi_{1}=q-1, \phi_{2}=q+1, \phi_{8}^{\prime}=q+\sqrt{2 q}+1, \phi_{8}^{\prime \prime}=q-\sqrt{2 q}+1, \phi_{8}=q^{2}+1, \phi_{12}=q^{2}-q+1, \\
\phi_{24}^{\prime}=q^{2}+q \sqrt{2 q}+q+\sqrt{2 q}+1=\left|T_{2}\right|, \phi_{24}^{\prime \prime}=q^{2}-q \sqrt{2 q}+q-\sqrt{2 q}+1=\left|T_{1}\right|,
\end{gathered}
$$

and $\phi_{24}=q^{4}-q^{2}+1$, so that $\phi_{24}=\phi_{24}^{\prime} \phi_{24}^{\prime \prime}=\left|T_{1}\right| \cdot\left|T_{2}\right|$.

The notation of irreducible characters is as in [41]. Note that their parametrization is the same as in Hiss [28] (where $\xi_{j}$ is used for $\chi_{j}$ ).

9.1. Brauer characters. Note that we only need to deal with non-liftable irreducible Brauer characters as $\ell \neq p$. The set of such characters is denoted by $\operatorname{Irr}_{\ell}^{0}(G)$. Note that tori $T_{1}, T_{2}$ satisfy the assumption of Lemma 3.2. By Lemma 3.7, every character in $\operatorname{Irr}_{\ell}^{0}(G)$ is unipotent and constant on the $\ell^{\prime}$-elements of $T_{i}, i=1,2$. The result is trivial if the constant in question equals 0 (for $i \in\{1,2\}$ ), as in this case $\left.\phi\right|_{T_{i}}$ is a multiple of the regular character $\rho_{T_{i}}^{\text {reg }}$. So below we only consider the cases where $\phi(t) \neq 0$ for $1 \neq t \in T_{i}$. If $\ell$ divides $\left.\left|T_{i}\right|\right)$ then we write $T_{i}^{\prime}$ for the subgroup of $\ell^{\prime}$-elements of $T_{i}, i=1,2$.

Every irreducible Brauer character agrees on $\ell^{\prime}$-elements with some integral linear combination of ordinary characters. If an $\ell$-modular character $\tau$, say, agrees on $\ell^{\prime}$-elements with $\sum a_{i} \chi_{i}$, where the $\chi_{i}{ }^{\prime}$ 's are ordinary characters, we simply write $\tau=\sum a_{i} \chi_{i}$. (or $\tau=\ell^{\prime} \sum a_{i} \chi_{i}$.)

Lemma 9.7. Let $\phi \in \operatorname{Irr}_{\ell}^{0}(G), T \in\left\{T_{1}, T_{2}\right\}$, and $t \in T$. Then either $\phi(t) \geq 0$, or $-\phi(t)<$ $q\left(q^{3}+q^{2}+1\right) / 8$, or $\ell=3, q=8$ and $T=T_{1}$.

Proof. (i) We start with primes $\ell$ for which Sylow $\ell$-subgroups are cyclic. This means that $\ell$ divides either $q^{2}-q+1$ or $\left|T_{1}\right|$ or $T_{2}$. These are the cases (a), (b), (c) below.

(a) Suppose that $\ell \mid\left(q^{2}-q+1\right)$. Then, by Hiss [28, Theorem 4.5], $\left|\operatorname{Irr}_{\ell}^{0}(G)\right|=2$, and for $\phi_{1}, \phi_{2} \in \operatorname{Irr}_{\ell}^{0}(G)$ we have $\phi_{1}=\chi_{11}-1_{G}, \phi_{2}=\chi_{4}-\chi_{11}-\chi_{19}-\chi_{20}+1_{G}$. Then $\phi_{1}(t)=-1$ and $\phi_{2}(t)=0$ for every $1 \neq t \in T_{1} \cup T_{2}$. So the result follows from Lemma 9.6 .

(b) Let $\ell$ divide $\left|T_{1}\right|$. Then, by Hiss [28, Theorem 4.7], $\left|\operatorname{Irr}_{\ell}^{0}(G)\right|=4$, and we have $\phi_{1}=$ $\chi_{10}-1_{G}, \phi_{2}=\chi_{7}-\chi_{5}-\chi_{15}-\chi_{19}, \phi_{3}=\chi_{8}-\chi_{6}-\chi_{16}-\chi_{20}$ and $\phi_{4}=\chi_{4}-\phi_{1}-\phi_{2}-\phi_{3}$. Then for every $1 \neq t \in T_{2}$ we have $\phi_{1}(t)=-1, \phi_{2}(t)=\phi_{3}(t)=1$ and $\phi_{4}(t)=0$.

In turn, for every $1 \neq t \in T_{1}^{\prime}$ we have $\phi_{1}(t)=-2, \phi_{2}(t)=\phi_{3}(t)=-4$, and $\phi_{4}(t)=11$. So the result follows from Lemma 9.6.

(c) Let $\ell$ divide $\left|T_{2}\right|$. Then, by Hiss [28, Theorem 4.6], $\left|\operatorname{Irr}_{\ell}^{0}(G)\right|=4$, and we have

$$
\phi_{1}=\chi_{9}-1_{G}, \phi_{2}=\chi_{7}-\chi_{5}, \phi_{3}=\chi_{8}-\chi_{6}, \phi_{4}=\chi_{4}-\chi_{9}+1_{G} .
$$

Then for every $1 \neq t \in T_{1}$ we have $\phi_{1}(t)=-1, \phi_{2}(t)=\phi_{3}(t)=-2, \phi_{4}(t)=2$, and for every $1 \neq t \in T_{2}^{\prime}$ we have $\phi_{1}(t)=-2, \phi_{2}(t)=\phi_{3}(t)=2$, and $\phi_{4}(t)=3$. So the result follows from Lemma 9.6.

(ii) Next we consider the cases where Sylow $\ell$-subgroups are not cyclic. Our main reference is [26]. Our ordering of the unipotent characters is as in [28] and [41], which is different from those in [26. So we indicate by arrows the correspondence of our characters to those in [26]:

$$
\begin{array}{r}
1_{G}=\chi_{1} \rightarrow \chi_{1}, \chi_{2} \rightarrow \chi_{4}, \chi_{3} \rightarrow \chi_{18}, \chi_{4} \rightarrow \chi_{21}, \chi_{5} \rightarrow \chi_{2}, \chi_{6} \rightarrow \chi_{3}, \chi_{7} \rightarrow \chi_{19}, \\
\chi_{8} \rightarrow \chi_{20}, \chi_{9} \rightarrow \chi_{5}, \chi_{10} \rightarrow \chi_{6}, \chi_{11} \rightarrow \chi_{7}, \chi_{12} \rightarrow \chi_{8}, \chi_{13} \rightarrow \chi_{9}, \chi_{14} \rightarrow \chi_{10}, \\
\chi_{15} \rightarrow \chi_{11}, \chi_{16} \rightarrow \chi_{12}, \chi_{17} \rightarrow \chi_{13}, \chi_{18} \rightarrow \chi_{14}, \chi_{19} \rightarrow \chi_{15}, \chi_{20} \rightarrow \chi_{16}, \chi_{21} \rightarrow \chi_{17} .
\end{array}
$$

(d) $\ell \mid\left(q^{2}-1\right)$. Here $\left|\operatorname{Irr}_{\ell}^{0}(G)\right|=0$. 
(e) $3 \neq \ell \mid\left(q^{2}+1\right)$. Then $\operatorname{Irr}_{\ell}^{0}(G)=\left\{\phi_{i}: i=4,7,18,21\right\}$ in notation of [26]. We have $\phi_{4}=\chi_{2}-1_{G}$ so $\phi_{4}(t)=-1$ and for all $1 \neq t \in T_{1} \cup T_{2}$.

$\phi_{7}=\chi_{11}-\chi_{2}$ and $\phi_{18}=\chi_{3}-a \chi_{21}-\chi_{11}+\chi_{2}$, where $2 \leq a \leq\left(q^{2}-2\right) / 3$, see [26, Theorem 3.2]. Then $\phi_{7}(t)=\phi_{18}(t)=0$ for all $1 \neq t \in T_{1} \cup T_{2}$.

Furthermore, we have

$$
\phi_{21}=\chi_{4}-e \phi_{18}-d \chi_{21}-c \chi_{13}-b \chi_{12}-\phi_{7}-\phi_{4},
$$

where $1 \leq b \leq(q+\sqrt{2 q}) / 4,1 \leq c \leq(q-\sqrt{2 q}) / 4,2 \leq d \leq\left(q^{2}+2\right) / 3,2 \leq e \leq(q+2) / 2$, so $\phi_{21}(t)=2-c$ for all $1 \neq t \in T_{1}$ and $\phi_{21}(t)=2-b$ for all $1 \neq t \in T_{2}$. In both the cases $\phi_{21}(t)<q\left(q^{3}+q^{2}+1\right) / 8$, so the result follows by Lemma 9.6 .

(f) $\ell=3$. Then $\left(3, q^{4}-q^{2}+1\right)=1$ as $3 \mid\left(q^{2}-1\right)$. In this case there are 22 unipotent Brauer characters and $\operatorname{Irr}_{\ell}^{0}(G)=\left\{\phi_{5,1}, \phi_{i}: i \in\{4,7,8,10,15,18,21\}\right.$.

In this case the expressions of $\phi_{i}$ in terms of ordinary characters $\chi_{j}$ depend of parameters which are not determined in full but satisfy certain inequalities. This are

$$
\begin{gathered}
2 \leq a \leq q, 0 \leq b \leq(q+\sqrt{2 q}) / 4,0 \leq c \leq(q-\sqrt{2 q}) / 4,2 \leq d \leq q^{2}, 1 \leq e \leq(q+2) / 2, \\
0 \leq x_{7} \leq q / 2,0 \leq x_{8} \leq(q+3 \sqrt{2 q}+4) / 12,0 \leq x_{10} \leq(q-2) / 6,1 \leq x_{15} \leq(q+1) / 3, \\
0 \leq x_{18} \leq q(q-1), 1 \leq x_{21} \leq q^{3} .
\end{gathered}
$$

We have

$\phi_{4}=\chi_{2}-1_{G}$, so $\phi_{4}(t)=-1$ for $1 \neq t \in T_{1} \cup T_{2}$;

$\phi_{5,1}=\chi_{20}-\chi_{21}$, whence $\phi_{5,1}(t)=1$ for $1 \neq t \in T_{1} \cup T_{2}$.

$\phi_{8}=\chi_{12}-x_{8} \phi_{5,1}$. So $\phi_{8}(t)=1-x_{8}$ if $T=T_{1}$ and $-x_{8}$ if $T=T_{2}$. As $x_{8}<q\left(q^{3}+q^{2}+1\right) / 8$, Lemma 9.6 applies.

$\phi_{7}=\chi_{11}-1_{G}-\phi_{4}-x_{7} \phi_{5,1}=\chi_{11}-\chi_{2}-x_{7} \phi_{5,1}$. Note that $\chi_{11}(t)=0$ for $1 \neq t \in T_{1} \cup T_{2}$. So $\phi_{7}(t)=-x_{7}$, and $x_{7}<q\left(q^{3}+q^{2}+1\right) / 8$.

$\phi_{10}=\chi_{14}-\phi_{8}-x_{10} \phi_{5,1}$. Note that $\chi_{14}(t)=0$ for $1 \neq t \in T_{1} \cup T_{2}$. So $\phi_{10}(t)=-x_{8}-x_{10}$ if $t \in T_{2}$ and $1-x_{8}-x_{10}$ if $t \in T_{1}$. Here $x_{8}+x_{10}<q+(q-2) / 6<q\left(q^{3}+q^{2}+1\right) / 8$.

$\phi_{15}=\chi_{19}-x_{15} \phi_{5,1}$, where $1 \leq x_{15} \leq(q+1) / 3$. As $\chi_{19}(t)=1$ for $1 \neq t \in T_{1} \cup T_{2}$, we have $\phi_{15}=1-x_{15}$. As above, $x_{15}<q\left(q^{3}+q^{2}+q\right) / 8$ yields the result.

$\phi_{18}=\chi_{3}-\phi_{7}-x_{18} \phi_{5,1}-a \phi_{15}$. So $\phi_{18}(t)=x_{7}-x_{18}+a\left(x_{15}-1\right)$ for $1 \neq t \in T_{1} \cup T_{2}$. As $x_{18}+a<q\left(q^{3}+q^{2}+1\right) / 8$, the result follows from Lemma 9.6 .

$\phi_{21}=\chi_{4}-\phi_{4}-\phi_{7}-x_{21} \phi_{5,1}-b \phi_{8}-c \phi_{10}-d \phi_{15}-e \phi_{18}$. So $\phi_{21}(t)=2+x_{7}+d\left(x_{15}-1\right)-x_{21}-e\left(x_{7}-\right.$ $\left.x_{18}+a\left(x_{15}-1\right)\right)-b \phi_{8}(t)-c \phi_{10}(t)=2+(1-e) x_{7}+(d-a e) x_{15}-x_{21}+e x_{18}-a-d-b \phi_{8}(t)-c \phi_{10}(t)$. In addition, $\phi_{8}(t)=1-x_{8}$ if $T=T_{1}$ and $-x_{8}$ if $T=T_{2}$, and $\phi_{10}(t)=1-x_{8}-x_{10}$ if $1 \neq t \in T_{1}$ and $-\left(x_{8}+x_{10}\right)$ if $t \in T_{2}$. So $-b \phi_{8}(t)-c \phi_{10}(t)=-b\left(1-x_{8}\right)-c\left(1-x_{8}-x_{10}\right)=(b+c)\left(x_{8}-1\right)+c x_{10}$ if $1 \neq t \in T_{1}$, and $-b\left(-x_{8}\right)-c\left(-x_{8}-x_{10}\right)=(b+c) x_{8}+c x_{10}$ if $1 \neq t \in T_{2}$. So

$\phi_{21}(t)= \begin{cases}2-a-d+(1-e) x_{7}+(d-a e) x_{15}-x_{21}+e x_{18}+(b+c)\left(x_{8}-1\right)+c x_{10} & 1 \neq t \in T_{1}, \\ 2-a-d+(1-e) x_{7}+(d-a e) x_{15}-x_{21}+e x_{18}+(b+c) x_{8}+c x_{10} & 1 \neq t \in T_{2} .\end{cases}$

Therefore

$$
-\phi_{21}(t) \leq a+d+(e-1) x_{7}+(a e-d) x_{15}+x_{21} \leq q+q^{2}+\frac{q^{2}}{4}+\frac{q(q-2)(q+3)}{6}+q^{3}<2 q^{3} .
$$

If $q>8$ then $2 q^{3}<q\left(q^{3}+q^{2}+q\right) / 8$, so the result follows by Lemma 9.6 .

Let $q=8$. If $t \in T_{2}$ then

$$
-\phi_{21}(t) \cdot(|T|-1) \leq 688 \cdot 36=24768<q(q-1)\left(q^{3}+q^{2}+1\right) \sqrt{q / 2}=112 \cdot 577=64624,
$$

so Lemma 9.6 yields the result.

As $\phi_{21}$ is constant on $T_{1} \backslash\{1\}$, all non-trivial $|t|$-roots of unity are eigenvalue of $\phi_{21}(t)$. 
Remark 9.8. Observe that $\left|T_{1}\right|=109,\left|T_{2}\right|=37$. By [26, Corollary 4.2], $c=1$ for $q=8$. Then $-\phi_{21}(t) \leq-2+a+d+(e-1) x_{7}+(a e-d) x_{15}+x_{21} \leq-2+q+q^{2}+\frac{q^{2}}{2}+\frac{\left.q^{2}+2 q+4\right)(q+1)}{6}+q^{3}=$ $6+64+32+111+512=725$ and $-\phi_{21}(t)\left(\left|T_{1}\right|-1\right) \leq 78300$. The lower bound for $\operatorname{dim} \phi$ suggested in [56], see Lemma 9.5, is $q(q-1)\left(q^{3}+q^{2}+1\right) \sqrt{q / 2}$ for $q=8$ yields $\operatorname{dim} \phi \geq 64624$. Note that if $1_{T_{1}}$ is not a constituent of $\left.\phi\right|_{T_{1}}$ then $\operatorname{dim} \phi$ is a multiple of $\left|T_{1}\right|-1=108$. As 64624 is not a multiple of 108 , we conclude that $\operatorname{dim} \phi \geq 64692$. So if $t \in T_{1}$ then the question remains open.

Proof of Theorem 1.1. The result follows from Lemma 6.1 when $G={ }^{2} B_{2}(q)$, Lemma 6.2 when $G={ }^{2} G_{2}(q)$, Theorem 7.1 and Lemma 7.13 when $G=G_{2}(q)$, Theorem 8.1 when $G={ }^{3} D_{4}(q)$, and from Lemmas $9.2,9.3$, Proposition 9.4 and Remark 9.8 when $G={ }^{2} F_{4}(q)$.

\section{REFERENCES}

[1] H. Azad, M. Berry and G. Seitz, On the structure of parabolic subgroups, Comm. Algebra 18(1990), $551-562$.

[2] M. Broué and G. Malle, Generalized Harish-Chandra theory, in: 'Representations of reductive groups', Ed. R.W. Carter and M. Geck, Cambridge Univ. Press, 1998.

[3] R. Burkhardt, Über die Zerlegungszahlen der Suzukigruppen Sz(q), J. Algebra 59 (1979), 421-433.

[4] R. Carter, "Finite Groups of Lie type: Conjugacy Classes and Complex Characters", Wiley, Chichester, 1985.

[5] B. Chang and R. Ree, The characters of $G_{2}(q)$, In: 'Symposia Math. XIII, Acad. Press, London, 1974', 395-413.

[6] J.H. Conway, R.T. Curtis, S.P. Norton, R.A. Parker and R.A. Wilson, ATLAS of Finite Groups, Clarendon Press, Oxford, 1985.

[7] B. Cooperstein, Maximal subgroups of $G_{2}\left(2^{n}\right)$, J. Algebra 70 (1981), 23-36.

[8] D.A. Craven, Representation Theory of Finite Groups: a Guidebook, Springer, 2019.

[9] D.I. Deriziotis and G.O. Michler, Character table and blocks of finite simple triality groups ${ }^{3} D_{4}(q)$, Trans. Amer. Math. Soc. 303 (1987), 39-70.

[10] F. Digne and J. Michel, "Representations of Finite Groups of Lie Type", London Mathematical Society Student Texts 21, Cambridge University Press, 1991.

[11] L. Di Martino and A. Zalesskii, Minimum polynomials and lower bounds for eigenvalue multiplicities of prime-power order elements in representations of quasi-simple groups, J. Algebra 243 (2001), 228-263; Corrigendum, J. Algebra 296 (2006), 249-252.

[12] O. Dudas, A note on decomposition numbers for groups of Lie type of small rank, J. Algebra 388 (2013), 364-373.

[13] O. Dudas, Coxeter Orbits and Brauer Trees II, Intern. Math. Research Notices, no. 15 (2014), 4100-4123.

[14] H. Enomoto, The characters of the finite Chevalley group $G_{2}(q), q=3^{f}$, Japan. J. Math. 2 (1976), 191247.

[15] H. Enomoto and H. Yamada, The characters of $G_{2}\left(2^{n}\right)$, Japan. J. Math. 12 (1986), 325-377.

[16] W. Feit, "The Representation Theory of Finite Groups", North-Holland, Amsterdam, 1982.

[17] M. Geck, Irreducible Brauer characters of the 3-dimensional special unitary groups in non-describing characteristic, Comm. Algebra 18 (1990), 563-584.

[18] M. Geck, Generalized Gelfand-Graev characters for Steinberg's triality groups and their applications, Comm. Algebra 19 (1991), 3249-3269.

[19] M. Geck, Basic sets of Brauer characters of finite groups of Lie type II, J. London Math. Soc. 47 (1993), 255-268.

[20] M. Geck and G. Hiss, Basic sets of Brauer characters of finite groups of Lie type, J. reine angew. Math. 418 (1991), 173-188.

[21] D. Gorenstein and R. Lyons, The local structure of finite groups of characteristic 2 type, Memoirs Amer. Math. Soc., vol.42, no. 276, Amer. Math. Soc. Providence, 1983.

[22] D. Gorenstein, R. Lyons and R. Solomon, The classification of the finite simple groups, Math. Surveys and Monographs vol. 40, no. 3, Amer. Math. Soc. Providence, 1998.

[23] R.M. Guralnick, K. Magaard, J. Saxl, and Pham Huu Tiep, Cross characteristic representations of odd characteristic symplectic groups and unitary groups, J. Algebra 257(2002), 291-347; Addendum, J. Algebra 299 (2006), 443-446.

[24] R.M. Guralnick and Pham Huu Tiep, Low dimensional representations of special linear groups in cross characteristics, Proc. London Math. Soc. 78 (1999), 116-138.

[25] F. Himstedt, On the decomposition numbers of the Ree groups ${ }^{2} F_{4}\left(q^{2}\right)$ in non-defining characteristic, preprint 
[26] F. Himstedt, On the 2-decomposition numbers of Steinberg's triality groups ${ }^{3} D_{4}(q)$, $q$ odd, J. Algebra 309 (2007), 569-593.

[27] G. Hiss, Regular and semisimple blocks of finite reductive groups, J. London Math. Soc. 41 (1990), 63-68.

[28] G. Hiss, The Brauer trees for the Ree groups, Comm. Algebra 19 (1991), 871-888.

[29] G. Hiss, "Zerlegungszahlen endlicher Gruppen vom Lie-Typ in nicht-definierender Characteristik", Habilitationsschrift, Lehrstuhl D für Mathematik, RWTH Aachen, Germany, 1993.

[30] G. Hiss, Hermitian function fields, classical unitals, and representations of 3-dimensional unitary groups, Indag. Math. (N.S.) 15 (2004), 223-243.

[31] G. Hiss and G. Malle, Low dimensional representations of special unitary group, J. Algebra 236 (2001), $745-767$.

[32] B. Huppert and N. Blackburn, "Finite Groups II", Springer-Verlag, Berlin etc., 1982.

[33] C. Jansen, K. Lux, R.A. Parker, and R.A. Wilson, 'An ATLAS of Brauer Characters', Oxford University Press, Oxford, 1995.

[34] W. Kantor, Subgroups of classical groups generated by long root elements, Trans. Amer. Math. Soc. 248 (1979), 347-379.

[35] P. Kleidman, The maximal subgroups of the Chevalley groups $G_{2}(q)$, with $q$ odd, the Ree groups ${ }^{2} G_{2}(q)$, and their automorphism groups, J. Algebra 117 (1988), 30-71.

[36] P. Kleidman, The maximal subgroups of the Steinberg triality groups ${ }^{3} D_{4}(q)$ and of their automorphism groups, J. Algebra 115 (1988), 182-199.

[37] V.V. Korableva, On chief factors of parabolic subgroups of special finite simple groups of exceptional Lie type, Siber. Math. J. 58 (2017), 1034-1041.

[38] P. Landrock and G. Michler, Principal 2-blocks of the simple groups of Ree type, Trans. Amer. Math. Soc. 260 (1980), 83-111.

[39] M. Liebeck, J. Saxl and G. Seitz, Subgroups of maximal rank in finite exceptional groups of Lie type, Proc. London Math. Soc. (3) 65 (1992), 297-325.

[40] F. Lübeck, Small degree representations of finite Chevalley groups in defining characteristic, LMS J. Comput. Math. 4 (2001), 135-169.

[41] G. Malle, Die unipotenten Charaktere von ${ }^{2} F_{4}\left(q^{2}\right)$, Comm. Algebra, 18 (1990), 2361-2381.

[42] G. Malle and D. Testerman, Linear algebraic groups and finite groups of Lie type, Cambridge Univ. Press, Cambridge, 2011.

[43] G. McNinch, Semisimple modules for finite groups of Lie type, J. London Math. Soc. (2) 60 (1999), 771-792.

[44] G. Navarro, Characters and blocks of finite groups, Cambridge Univ. Press, Cambridge, 1998.

[45] T. Okuyama and K. Waki, Decomposition numbers of $S U\left(3, q^{2}\right)$, J. Algebra 255 (2002), 258-270.

[46] M. Pellegrini and A.E. Zalesski, Irreducible characters of finite simple groups constant on $p$-singular elements, Rendiconti del Seminario Matematico della Universitá di Padova 136 (2016), 35-50.

[47] A. Premet and I. Suprunenko, Quadratic modules for Chevalley groups over fields of odd characteristics, Math. Nachr. 110 (1983), 65-96.

[48] G.M. Seitz and A.E. Zalesskiǔ, On the minimal degrees of projective representations of the finite Chevalley groups II, J. Algebra 158 (1993), 233-243.

[49] J. Shamash, Brauer trees for blocks of cyclic defect in the groups $G_{2}(q)$ for primes dividing $q^{2} \pm q+1, J$. Algebra 123 (1989), 378-396.

[50] J. Shamash, Blocks and Brauer trees for the groups $G_{2}\left(2^{k}\right), G_{2}\left(3^{k}\right)$, Comm. Algebra 20 (1992), $1375-1387$.

[51] P. Sin, Extensions of simple modules for $S L_{3}\left(2^{n}\right)$ and $S U_{3}\left(2^{n}\right)$, Proc. London Math. Soc. (3) 65 (1992), 265-296.

[52] T. Springer and R. Steinberg, Conjugacy classes, In: A. Borel et al,'Seminar on algebraic groups and related finite groups', Springer-Verlag, Berlin, 1970.

[53] I. D. Suprunenko, The minimal polynomials of unipotent elements in irreducible representations of the classical groups in odd characteristic, Memoirs Amer. Math. Soc. 200 (2009), no. 939, 1-154.

[54] Pham Huu Tiep and A.E. Zalesski, Real conjugacy classes in algebraic groups and finite groups of Lie type, J. Group Theory 8 (2005), 291-315.

[55] Pham Huu Tiep and A.E. Zalesski, Hall-Higman type theorems for semisimple elements of finite classical group, Proc. London math. Soc. (3) 97 (2008), 623-668.

[56] Pham Huu Tiep, Finite groups admitting grassmannian 4-designs, J. Algebra 306 (2006), 227-243.

[57] K. Waki, Decomposition numbers of $S U\left(3, q^{2}\right)$, J. Algebra 255 (2002), 258-270.

[58] H. Ward, On Ree's series of simple groups, Trans. Amer. Math. Soc. 121 (1966), 62-89.

[59] A.E. Zalesskir, The eigenvalue 1 of matrices of complex representations of finite Chevalley groups, Proc. Steklov Inst. of Math. 1991, issue 4, 109-119.

[60] A.E. Zalesskiǔ, Minimal polynomials and eigenvalues of $p$-elements in representations of quasi-simple groups with a cyclic Sylow p-subgroup, J. London Math. Soc. 59 (1999), 845-866.

[61] A.E. Zalesski, The number of distinct eigenvalues of elements in finite linear groups, J. London Math. Soc. 74 (2006), 361-378. 
[62] A.E. Zalesski, Minimal polynomials of the elements of prime order in complex irreducible representations of quasi-simple groups, J. Algebra 320 (2008), 2496-2525.

Department of Mathematics, Rutgers University, Piscataway, NJ 08854, U.S.A.

Email address: tiep@math.rutgers.edu

Department of Physics, Mathematics and Informatics, National Academy of Sciences of Belarus, 66 Prospekt Nezavisimosti, Minsk, Belarus

Email address: a.zalesskii@uea.ac.uk 